Research Article

\title{
Novel Dombi Aggregation Operators in Spherical Cubic Fuzzy Information with Applications in Multiple Attribute Decision-Making
}

\author{
Tehreem $\mathbb{D}^{1},{ }^{1}$ Amjad Hussain, ${ }^{1}$ and Ahmed Alsanad $\mathbb{D D}^{2}$ \\ ${ }^{1}$ Department of Mathematics, Quaid-i-Azam University Islamabad, Islamabad, Pakistan \\ ${ }^{2}$ STC's Artificial Intelligence Chair, Department of Information Systems, College of Computer and Information Sciences, \\ King Saud University, Riyadh 11451, Saudi Arabia
}

Correspondence should be addressed to Ahmed Alsanad; aasanad@ksu.edu.sa

Received 16 March 2021; Accepted 22 May 2021; Published 10 July 2021

Academic Editor: G. Muhiuddin

Copyright $\left({ }_{0} 2021\right.$ Tehreem et al. This is an open access article distributed under the Creative Commons Attribution License, which permits unrestricted use, distribution, and reproduction in any medium, provided the original work is properly cited.

\begin{abstract}
The notion of spherical fuzzy sets (SFSs) is one of the most effective ways to model the fuzzy information in decision-making processes. The sum of squares of membership, neutral, and nonmembership degrees in SFSs lies in the interval $[0,1]$ and accommodates more uncertainties. Henceforth, in this article, the idea of spherical cubic fuzzy sets (SCFSs) is introduced, which is the generalization of SFSs. Spherical cubic fuzzy set is the combination of spherical fuzzy sets and interval-valued spherical fuzzy sets. The membership, neutral, and nonmembership degrees in an SCFS are cubic fuzzy numbers (CFNs). Consequently, this set outperforms the pre-existing structures of fuzzy set theory. Moreover, some fundamental operations for the comparison of two spherical CFNs are defined such as score function and accuracy function. Further, several new operations through Dombi t-norm and Dombi t-conorms are characterized to get the best results during the decision criteria. Furthermore, spherical cubic fuzzy Dombi weighted averaging (SCFDWA), SCFD ordered weighted averaging (SCFDOWA), SCFD hybrid weighted averaging (SCFDHWA), SCFD weighted geometric (SCFDWG), SCFD ordered weighted geometric (SCFDOWG), and the SCFD hybrid weighted geometric (SCFDHWG) aggregated operators are discussed, and their characteristics are examined. In addition, some of the operational laws of these operators are defined. Also, a decision-making approach based on these operators is proposed. Since the proposed methods and operators are the generalizations of the existing methods and operators, therefore, these techniques produce more general, accurate, and precise results as compared with existing ones. Finally, a descriptive example is given in order to describe the validity, practicality, and effectiveness of the proposed methods.
\end{abstract}

\section{Introduction}

Multiattribute group decision-making is an investigation of recognizing and choosing the alternatives depending upon the values and priorities of decision makers. Settling on a chance infers that there are alternative decisions to be considered. In such a case, we do not need to recognize the same number of these options as could be allowed to select the best possibility to attain our objectives, targets, or expectations. Zadeh in [1] presented the idea of fuzzy set theory and logic. In the fuzzy set theory, he just examined the membership grade, known as the membership degree. Moreover, Zadeh established the application of fuzzy sets
(FSs) in various fields like design, software engineering, and information technology. After numerous uses of FSs, Atanassov [2] saw that there are several deficiencies in FSs so he presented the idea of intuitionistic fuzzy sets (IFSs). Each element in an IFS is described by a pair of mappings, and each of these mappings is categorized by a membership and a nonmembership grade. The IFS was further extended by Yager and Yager $[3,4]$ who developed the idea of Pythagorean fuzzy sets (PyFSs) by adding constraints on the membership and nonmembership grades as the aggregate of the squares of membership and nonmembership grades must not exceed by 1 . In $[5,6]$, the new approach of decision-making using the concept of picture fuzzy numbers 
was presented and further it was extended for picture fuzzy linguistic sets.

An IFS and a PyFS have been effectively implemented in various fields, but in numerous circumstances, these theories fail, for instance, in vote casting, human judgements include more responses like yes, no, abstain, and refusal. In voting station, the chamber issues forms for the applicants. The voting outcomes are distributed into four categories, and the results are as follows: vote in favor, abstain or neutral votes, vote in opposition, and refuse to vote. Here, abstain means blank voting form, i.e., nobody gave the vote in favor or against. Refusal of vote means that a person refuses to give the vote. The applicant is viewed as effective in the light of the fact that the quantity of supportive papers is greater than the vote in opposition. In these sorts of situations where the abstinence and rejection occur, the concepts of FSs and IFSs fail to be applied. Hence, the concept of picture fuzzy set (PFS) is presented by Cuong et al. [7-9], that is, the expanded form of the FSs and IFSs. PFS gives three membership grades known as positive membership grade, neutral membership grade, and negative membership grade correspondingly. The issue of voting criteria successfully resolved by picture fuzzy set theory, and this theory is further applied in various fields. By making use of those operations for PFN, plenty of working has been performed by the research workers, combined with aggregated methodology, multiattribute group decision, and information measures. From this overview, it is commented that neither FS nor IFS and PFS theory are useful to tackle the vague conflicting information. For example, if an individual gives their inclination about the item as far as indeed is, no is, and abstained is, at that point, we see that accordingly, IFSs and PFSs may not be able to tackle such circumstances. To overcome these complications, many researchers [10] presented the notion of spherical fuzzy set (SFS) by the additional constraints, i.e., the sum of the squares must not exceed. According to above situation, we see, thus, SFS is the generalization of PFS and IFS to cope with the problems in making decision. Fahmi et al. [11] gave a new idea of trapezoidal cubic fuzzy numbers and their applications in multiattribute group decision making. Garg [12] presented the idea of picture fuzzy aggregation operators and further discussed its applications. Ashraf et al. [13] introduced the collection of spherical weighted aggregated operations for resolving multiattribute decision-making problems under the spherical fuzzy sets. Gündogdu and Kahraman [14] presented the new idea of the spherical fuzzy TOPSIS method.

Dombi operations introduced in 1982 are an important complement to the existing operations. It is characterized by good flexibility for information aggregation. Many scholars presented the t-norm and $\mathrm{t}$-conorm Dombi operations in $[5,15]$ which have an inclination of fluctuation with the operation of parameters. For this preferred position, Lin et al. utilized the IFSs and combined them with Dombi operations and presented the concept of Dombi Bonferroni mean operator [16] using IFSs to resolve the issues in multiattribute group decision-making. Liu et al. [17] presented new concept of spherical fuzzy sets for Yunnan
Baiyao's R \& D project selectionproblem. In [18], Shi and Ye extended Dombi operation to neutrosophic cubic sets and utilized it in travelling decision-making approaches. To resolve the various issues in multiattribute group decision makers, Lu and Ye [19] firstly defined Dombi aggregated operations and linguistic cubic sets [20-23]. In [24], Jana et al. presented several Dombi bipolar fuzzy aggregated operations under the picture fuzzy data based on averaging, geometric, and various Dombi operations. Jana et al. [25] presented the idea of picture fuzzy Dombi aggregation operators and their applications in multiattribute decisionmaking process. Rafiq et al. [26] presented the cosine similarity measures of spherical fuzzy sets and their applications in decision-making. Seikh and Mandal [27] gave the idea of intuitionistic fuzzy Dombi aggregation operators and their applications. Wei et al. [28] presented the similarity measure of spherical fuzzy sets using the cosine function and their applications. Muneeza et al. [29, 30] gave the new idea of intuitionistic cubic fuzzy sets and their applications in supplier's selection and hydropower plant locations. In [31-33], many researchers introduced the idea of Pythagorean cubic fuzzy sets, which is the generalization of Pythagorean fuzzy sets and cubic sets and further discussed its application in multiattribute decision-making. Ayaz et al. $[34,35]$ introduced the idea of spherical cubic fuzzy sets and defined the various aggregation operators and their applications in decision-making.

In aggregated procedure, the significant method is to characterize the operational laws. The applications of SFSs require several new operations and aggregated operations to be developed. By maintaining the advantages of the SFS, we describe the collection of SFSs. Besides this, by using Dombi norms, the fundamental weighted geometric average operations have been characterized by utilizing the idea of cubic fuzzy set theory as spherical cubic fuzzy Dombi weighted average (SCFDWA), spherical cubic fuzzy Dombi ordered weighted average (SCFDOWA), spherical cubic fuzzy Dombi hybrid weighted average (SCFDHWA), spherical cubic fuzzy Dombi weighted geometric (SCFDWG), spherical cubic fuzzy Dombi ordered weighted geometric (SCFOWG), and spherical cubic fuzzy Dombi hybrid weighted geometric (SCFDHWG). We utilized these operations to propose the method for multiattribute group decision-making. At last, we enlighten the practicality of the proposed methods in the selection of spherical cubic fuzzy numbers. In order to get a fair decision during the process, some new operational laws by Dombi t-norm and t-conorm are defined in this manuscript. A new approach based on spherical cubic fuzzy set models via spherical cubic fuzzy Dombi aggregation operators is proposed. A method to deal with decision-making problems using spherical cubic Dombi weighted averaging, Dombi weighted geometric, and Dombi hybrid weighted aggregation operators is established. This model has a stronger capability than existing weighted averaging, weighted geometric, Einstein, logarithmic averaging, and logarithmic geometric aggregation operators for spherical cubic fuzzy information. This study presents the novel decision-making techniques to tackle the uncertainty in decision-making processes through proposed generalized 
structure of spherical cubic fuzzy set and well known Dombi norms.

The paper is designed in the following manner. In Section 2, we presented fundamental information of extended fuzzy sets. In Section 3, we have discussed the idea of spherical cubic fuzzy set and various aggregation operators. The Dombi aggregation operations are introduced in Section 4. Section 5 discusses the applications of the proposed method, and some numerical applications are given in Section 6. In Section 7, analysis with the suggested Dombi aggregated operations is carried out, and finally we conclude our work in Section 8.

\section{Preliminaries}

We support the reader's interpretation of the standard definitions and outcomes of the spherical fuzzy set theory, but to make this work more introspective, basic ideas used in the literature are described, and we note a portion of the idea and findings used in the rest of the work.

Definition 1 (see [30]). Let $X$ be the universe of discourse; a fuzzy intuitionistic cubic set $I_{\widetilde{x}}$ on $X$ is presented as

$$
I_{\tilde{x}}=\left\{\left\langle\left[\widehat{u}^{-}, \widehat{u}^{+}\right], \widehat{\alpha}\right\rangle,\left\langle\left[\widehat{w}^{-}, \widehat{w}^{+}\right], \widehat{\theta}\right\rangle \mid \tilde{x} \in X\right\},
$$

where $\left\langle\left[\widehat{u}^{-}, \widehat{u}^{+}\right], \widehat{\alpha}\right\rangle$ and $\left\langle\left[\widehat{w}^{-}, \widehat{w}^{+}\right], \widehat{\theta}\right\rangle$ are known as membership and nonmembership of $I_{\tilde{x}}$, which satisfy the condition that $\sup \left[\widehat{u}^{-}, \widehat{u}^{+}\right]+\sup \left[\widehat{w}^{-}, \widehat{w}^{+}\right] \leq 1$ and $\widehat{\alpha}+\hat{\theta} \leq 1$.

Definition 2 (see [24]). Let $X$ be the universe of discourse; a fuzzy Pythagorean cubic set $P_{\tilde{x}}$ on $X$ is presented as

$$
P_{\widetilde{x}}=\left\{\left\langle\left[\widehat{u}^{-}, \widehat{u}^{+}\right], \widehat{\alpha}\right\rangle,\left\langle\left[\left[\widehat{w}^{-}, \widehat{w}^{+}\right], \widehat{\theta}\right\rangle\right| \widetilde{x} \in X\right\},
$$

where $\left\langle\left[\widehat{u}^{-}, \widehat{u}^{+}\right], \widehat{\alpha}\right\rangle$ and $\left\langle\left[\widehat{w}^{-}, \widehat{w}^{+}\right], \widehat{\theta}\right\rangle$ are known as membership and nonmembership of $I_{\tilde{x}}$, which satisfy the condition that $\left(\sup \left[\widehat{u}^{-}, \widehat{u}^{+}\right]\right)^{2}+\left(\sup \left[\widehat{w}^{-}, \widehat{w}^{+}\right]\right)^{2} \leq 1$ and $\widehat{\alpha}^{2}+\widehat{\theta}^{2} \leq 1$.

Definition 3 (see [10]). Let $X$ be the universe of discourse; a spherical fuzzy set $S_{\widetilde{x}}$ on $X$ is presented as

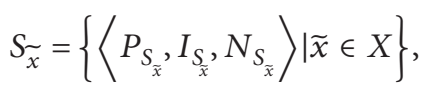

where $P_{S_{\tilde{x}}}: X \longrightarrow \theta, I_{S_{\tilde{x}}}: X \longrightarrow \theta$, and $N_{S_{\tilde{x}}}: X \longrightarrow \theta$ are known as membership, neutral, and nonmembership degrees, respectively, under the specific condition $0 \leq P_{S_{\tilde{x}}}+I_{S_{\widetilde{x}}}+N_{S_{\tilde{x}}} \leq 1$, and the triplet $S_{\tilde{x}}=\left\langle P_{S_{\widetilde{x}}} I_{S_{\widetilde{x}}}, N_{S_{x}}\right\rangle$ is called the spherical fuzzy numbers.

\section{Characteristics of Spherical Cubic Fuzzy Sets}

The definition of the spherical cubic fuzzy set (SCFS) and its operations are presented in this section. The extension of the spherical fuzzy set is the spherical cubic fuzzy set. The composition of the SCFS defends elements that met or did not fulfill the requirement for values to be from 0 to 1 .

The spherical cubic fuzzy set is a direct generalization of the cubic fuzzy set of Pythagoras and the cubic fuzzy set of images. When the pythagorean cubic fuzzy set (PyCFS) and image cubic fuzzy set both could not cope with the situation, a fascinating situation grows. There is a need to describe the concept of a spherical cubic fuzzy set to solve this situation in this way. The principle of the distinction between PyCFSs and SCFSs is that we research the neutral membership in SCFSs, where it does not in PyCFSs. The association of positive, neutral, and negative degrees of an object is defined in the closed unit interval in image cubic fuzzy, but the sum of positive, neutral, and negative degrees of the object is greater than 1. In this situation, we used spherical cubic fuzzy sets.

Each feature of the intuitionist cubic fuzzy set (ICFS) consists of a membership function and a nonmembership function in which the function of membership is cubic fuzzy set and cubic fuzzy set is also nonmembership. We then generalize (ICFS) and describe a fresh definition of the spherical cubic fuzzy set (SCFS) consisting of membership function, neutral membership function, and nonmembership function in which the function of membership is cubic fuzzy set, cubic fuzzy set is the function of neutral membership, and cubic fuzzy set is also nonmembership. The issue is addressed by SCFS in all current systems such as ICFS, PyCFS, and PCFS. So, the SCFS is the generalization of the entire system that exists.

Definition 4 (see [34]). Let $X$ be the universe of discourse, then the spherical cubic fuzzy set $\widetilde{S}_{\widetilde{x}}$ in $X$ is defined as

$$
\breve{S}_{\widetilde{x}}=\left\{x,\left\langle\left[\widehat{u}^{-}, \widehat{u}^{+}\right], \widehat{\alpha}\right\rangle,\left\langle\left[\widehat{v}^{-}, \widehat{v}^{+}\right], \widehat{\beta}\right\rangle,\left\langle\left[\widehat{w}^{-}, \widehat{w}^{+}\right], \widehat{\theta}\right\rangle \mid x \in X\right\},
$$

where $\left\langle\left[\widehat{u}^{-}, \widehat{u}^{+}\right], \widehat{\alpha}\right\rangle$ represents the membership degree, $\left\langle\left[\widehat{v}^{-}, \widehat{v}^{+}\right], \widehat{\beta}\right\rangle$ represents neutral, and $\left\langle\left[\widehat{w}^{-}, \widehat{w}^{+}\right], \widehat{\theta}\right\rangle$ represents the nonmembership degree where $\left[\widehat{u}^{-}, \widehat{u}^{+}\right] \subseteq[0,1]$, and $\left[\widehat{v}^{-}, \widehat{v}^{+}\right] \subseteq[0,1]$, and $\left[\widehat{w}^{-}, \widehat{w}^{+}\right] \subseteq[0,1]$ and $\hat{\alpha}: X \longrightarrow$ $[0,1], \quad \widehat{\beta}: X \longrightarrow[0,1]$, and $\widehat{\theta}: X \longrightarrow[0,1]$ under the condition

$$
\begin{array}{r}
\operatorname{Sup}\left[\widehat{u}^{-}, \widehat{u}^{+}\right]+\operatorname{Sup}\left[\widehat{v}^{-}, \widehat{v}^{+}\right]+\operatorname{Sup}\left[\left[\widehat{w}^{-}, \widehat{w}^{+}\right] \leq 1\right. \\
\widehat{\alpha}+\widehat{\beta}+\widehat{\theta} \leq 1 .
\end{array}
$$

We called $\left(\left\langle\left[\widehat{u}^{-}, \widehat{u}^{+}\right], \widehat{\alpha}\right\rangle,\left\langle\left[\widehat{v}^{-}, \widehat{v}^{+}\right], \widehat{\beta}\right\rangle,\left\langle\left[\widehat{w}^{-}, \widehat{w}^{+}\right], \widehat{\theta}\right\rangle\right)$ as spherical cubic fuzzy numbers (SCFNs) denoted by $S_{\widetilde{x}} . S_{\tilde{x}}=$ $\left(\left\langle\left[\widehat{u}^{-}, \widehat{u}^{+}\right], \widehat{\alpha}\right\rangle,\left\langle\left[\widehat{v}^{-}, \widehat{v}^{+}\right], \widehat{\beta}\right\rangle,\left\langle\left[\widehat{w}^{-}, \widehat{w}^{+}\right], \widehat{\theta}\right\rangle\right)$ which is known as spherical cubic fuzzy numbers (SCFNs).

$$
\breve{S}_{\widetilde{x}}=\left(\left\langle\left[\widehat{u}^{-}, \widehat{u}^{+}\right], \widehat{\alpha}\right\rangle,\left\langle\left[\widehat{v}^{-}, \widehat{v}^{+}\right], \widehat{\beta}\right\rangle,\left\langle\left[\widehat{w}^{-}, \widehat{w}^{+}\right], \widehat{\theta}\right\rangle\right) .
$$

Definition 5 (see [34]). Let two SCFNs be $\breve{S}_{\widetilde{x}_{1}}=\left\{\left\langle\left[\widehat{u}_{1}^{-}\right.\right.\right.$, $\left.\left.\left.\widehat{u}_{1}^{+}\right], \widehat{\alpha}_{1}\right\rangle,\left\langle\left[\widehat{v}_{1}^{-}, \widehat{v}_{1}^{+}\right], \widehat{\beta}_{1}\right\rangle,\left\langle\left[\widehat{w}_{1}^{-}, \widehat{w}_{1}^{+}\right], \widehat{\theta}_{1}\right\rangle \mid x \in X\right\}$ and $S_{\widetilde{x}_{2}}=$ $\left\{\left\langle\left[\widehat{u}_{2}^{-}, \widehat{u}_{2}^{+}\right], \widehat{\alpha}_{2}\right\rangle,\left\langle\left[\widehat{v}_{2}^{-}, \widehat{v}_{2}^{+}\right], \widehat{\beta}_{2}\right\rangle,\left\langle\left[\widehat{w}_{2}^{-}, \widehat{w}_{2}^{+}\right], \widehat{\theta}_{2}\right\rangle \mid x \in X\right\} \quad$ and $\gamma>0$ be any constant. Then, the operations are defined as follows:
(1) $\breve{S}_{\widetilde{x}_{1}}=\breve{S}_{\widetilde{x}_{2}}$ iff $\breve{S}_{\widetilde{x}_{1}} \subseteq \breve{S}_{\widetilde{x}_{2}}$ and $\breve{S}_{\widetilde{x}_{2}} \subseteq \breve{S}_{\widetilde{x}_{1}}$
(2) $\breve{S}_{\widetilde{x}_{1}} \cup \breve{S}_{\widetilde{x}_{2}}=\left\{\begin{array}{c}x,\left(r\left(\max \left[\hat{u}_{1}^{-}, \hat{u}_{1}^{+}\right],\left[\hat{u}^{-}, \widehat{u}_{2}^{+}\right]\right), \min \left(\widehat{\alpha}_{1}^{-}, \widehat{\alpha}_{1}^{+}\right)\right), \\ \left(r \min \left(\left[\hat{v}_{1}^{-}, \widehat{v}_{1}^{+}\right],\left[\hat{v}_{2}^{-}, \widehat{v}_{2}^{+}\right]\right), \max \left(\widehat{\beta}_{1}, \widehat{\beta}_{2}\right)\right), \\ \left(r \min \left(\left[\widehat{w}_{1}^{-}, \widehat{w}_{1}^{+}\right],\left[\hat{w}_{2}^{-}, \widehat{w}_{2}^{+}\right]\right), \max \left(\widehat{\theta}_{1}, \widehat{\theta}_{2}\right)\right)\end{array}\right\}$ 
(3) $\breve{S}_{\widetilde{x}_{1}} \cap \breve{S}_{\widetilde{x}_{2}}=\left\{\begin{array}{c}x,\left(r\left(\min \left[\widehat{u}_{1}^{-}, \widehat{u}_{1}^{+}\right],\left[\widehat{u}_{u}^{-}, \widehat{u}_{2}^{+}\right]\right), \max \left(\widehat{\alpha}_{1}^{-}, \widehat{\alpha}_{1}^{+}\right)\right), \\ \left(r \min \left(\left[\widehat{v}_{1}^{-}, \widehat{v}_{1}^{+}\right],\left[\hat{v}_{2}^{-}, \hat{v}_{2}^{+}\right]\right), \max \left(\widehat{\beta}_{1}, \widehat{\beta}_{2}\right)\right), \\ \left(r \max \left(\left[\widehat{w}_{1}^{-}, \widehat{w}_{1}^{+}\right],\left[\widehat{w}_{2}^{-}, \widehat{w}_{2}^{+}\right]\right), \min \left(\widehat{\theta}_{1}, \widehat{\theta}_{2}\right)\right)\end{array}\right\}$

(4) $\breve{S}_{x}^{c}=\left\{x,\left\langle\left[\widehat{w}^{-}, \widehat{w}^{+}\right], \widehat{\theta}\right\rangle,\left\langle\left[\widehat{v}^{-}, \widehat{v}^{+}\right], \widehat{\beta}\right\rangle,\left\langle\left[\widehat{u}^{-}, \widehat{u}^{+}\right], \widehat{\alpha}\right\rangle\right\}$

Definition 6 (see [34]). Let two SCFNs be $\breve{S}_{\widetilde{x}_{1}}=\left\{x,\left\langle\left[\widehat{u}_{1}^{-}\right.\right.\right.$, $\left.\left.\left.\widehat{u}_{1}^{+}\right], \widehat{\alpha}_{1}\right\rangle,\left\langle\left[\widehat{v}_{1}^{-}, \widehat{v}_{1}^{+}\right], \widehat{\beta}_{1}\right\rangle,\left\langle\left[\widehat{w}_{1}^{-}, \widehat{w}_{1}^{+}\right], \widehat{\theta}_{1}\right\rangle \mid x \in X\right\}$ and $S_{\widetilde{x}_{2}}=\{x$, $\left.\left\langle\left[\widehat{u}_{2}^{-}, \widehat{u}_{2}^{+}\right], \widehat{\alpha}_{2}\right\rangle,\left\langle\left[\widehat{v}_{2}^{-}, \widehat{v}_{2}^{+}\right], \widehat{\beta}_{2}\right\rangle,\left\langle\left[\widehat{w}_{2}^{-}, \widehat{w}_{2}^{+}\right], \widehat{\theta}_{2}\right\rangle \mid x \in X\right\}$. The operations of the SCFNS are defined as follows:

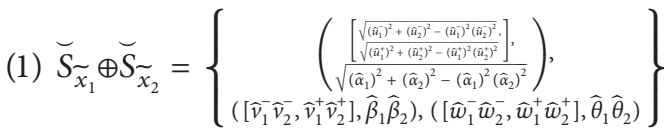

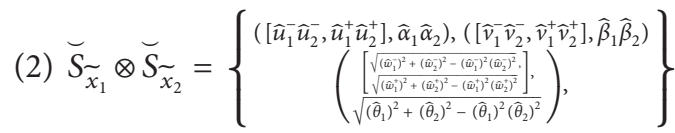

(3) $\gamma \cdot \breve{S}_{\widetilde{x}_{1}}=\left\{\begin{array}{c}\left(\sqrt{1-\left(1-\widehat{u}_{1}^{-}\right)^{\gamma}}, \sqrt{1-\left(1-\widehat{u}_{1}^{+}\right)^{\gamma}}\right] \\ \sqrt{1-\left(1-\widehat{\alpha}_{1}\right)^{\gamma}}\end{array}\right)$,

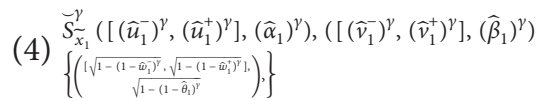

Definition 7 (see [35]). Let $\breve{S}_{\widetilde{x}_{1}}=\left\{x,\left\langle\left[\widehat{u}_{1}^{-}, \widehat{u}_{1}^{+}\right], \widehat{\alpha}_{1}\right\rangle,\left\langle\left[\widehat{v}_{1}^{-}\right.\right.\right.$, $\left.\left.\left.\widehat{v}_{1}^{+}\right], \widehat{\beta}_{1}\right\rangle,\left\langle\left[\widehat{w}_{1}^{-}, \widehat{w}_{1}^{+}\right], \widehat{\theta}_{1}\right\rangle\right\}$ and $S_{\widetilde{x}_{2}}^{\varkappa_{1}}=\left\{x,\left\langle\left[\widehat{u}_{2}^{-}, \widehat{u}_{2}^{+}\right], \widehat{\alpha}_{2}\right\rangle,\left\langle\left[\widehat{v}_{2}^{-}\right.\right.\right.$, $\left.\left.\left.\widehat{v}_{2}^{+}\right], \widehat{\beta}_{2}\right\rangle,\left\langle\left[\widehat{w}_{2}^{-}, \widehat{w}_{2}^{+}\right], \widehat{\theta}_{2}\right\rangle\right\}$ and $\gamma_{1}, \gamma_{2} \geq 0$ are any two constants, then we have the following properties:

(1) $\breve{S}_{\widetilde{x}_{1}} \oplus \breve{S}_{\widetilde{x}_{2}}=\breve{S}_{\widetilde{x}_{2}} \oplus \breve{S}_{\widetilde{x}_{1}}$

(2) $\breve{S}_{\widetilde{x}_{1}} \otimes \breve{S}_{\widetilde{x}_{2}}=\breve{S}_{\widetilde{x}_{2}} \otimes \breve{S}_{\widetilde{x}_{1}}$

(3) $\gamma_{1}\left(\breve{S}_{\widetilde{x}_{1}} \oplus \breve{S}_{\widetilde{x}_{2}}\right)=\gamma_{1} \breve{S}_{\widetilde{x}_{1}} \oplus \gamma_{1} \breve{S}_{\widetilde{x}_{2}}, \gamma_{1}>0$

(4) $\left(\breve{S}_{\widetilde{x}_{1}} \otimes \breve{S}_{\widetilde{x}_{2}}\right)^{\gamma_{1}}=\breve{S}_{\widetilde{x}_{1}}^{\gamma_{1}} \otimes \breve{S}_{\widetilde{x}_{2}}^{\gamma_{1}}, \gamma_{1}>0$

(5) $\gamma_{1} \breve{S}_{\widetilde{x}_{1}} \oplus \gamma_{2} \breve{S}_{\widetilde{x}_{1}}=\left(\gamma_{1}+\gamma_{2}\right) \breve{S}_{\widetilde{x}_{1}}, \gamma_{1}, \gamma_{2}>0$

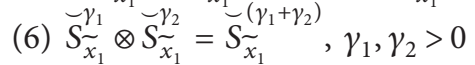

(7) $\left(\breve{S}_{\widetilde{x}_{1}} \oplus \breve{S}_{\widetilde{x}_{2}}\right) \oplus \breve{S}_{\widetilde{x}_{3}}=\breve{S}_{\widetilde{x}_{1}} \oplus\left(\breve{S}_{\widetilde{x}_{2}} \oplus \breve{S}_{\widetilde{x}_{3}}\right)$

(8) $\left(\breve{S}_{\widetilde{x}_{1}} \otimes \breve{S}_{\widetilde{x}_{2}}\right) \otimes \breve{S}_{\widetilde{x}_{3}}=\breve{S}_{\widetilde{x}_{1}} \otimes\left(\breve{S}_{\widetilde{x}_{2}} \otimes \breve{S}_{\widetilde{x}_{3}}\right)$

Definition 8 (see $[35])$. Let $\breve{S}_{\widetilde{x}}=\left(\left\langle\left[\widehat{u}^{-}, \widehat{u}^{+}\right], \widehat{\alpha}\right\rangle,\left\langle\left[\widehat{v}^{-}\right.\right.\right.$, $\left.\left.\left.\widehat{v}^{+}\right], \widehat{\beta}\right\rangle,\left\langle\left[\widehat{w}^{-}, \widehat{w}^{+}\right], \widehat{\theta}\right\rangle\right)$ SCFN; the score function $S_{c}\left(S_{\widetilde{x}}\right)$ is presented as

$$
S_{c}\left(\breve{S}_{\tilde{x}}\right)=\frac{\left(\widehat{u}^{-}+\widehat{u}^{+}+\widehat{\alpha}\right)^{2}+\left(\widehat{v}^{-}+\widehat{v}^{+}+\widehat{\beta}\right)^{2}-\left(\widehat{w}^{-}+\widehat{w}^{+}+\widehat{\theta}\right)^{2}}{9} .
$$

Here, $S_{c}\left(\breve{S}_{\widetilde{x}}\right) \in[-1,1]$.

Definition 9. An accuracy function $A\left(\breve{S}_{\widetilde{x}}\right)$ is presented as

$$
A\left(\breve{S}_{\widehat{x}}\right)=\frac{\left(\widehat{u}^{-}+\widehat{u}^{+}+\widehat{\alpha}\right)^{2}+\left(\widehat{v}^{-}+\widehat{v}^{+}+\widehat{\beta}\right)^{2}+\left(\widehat{w}^{-}+\widehat{w}^{+}+\widehat{\theta}\right)^{2}}{9}
$$

Here, $A\left(\breve{S}_{\widetilde{x}}\right) \in[0,1]$.

Definition 10. For any SCFNs, $\breve{S}_{\widetilde{x}_{p}}=\left\langle\breve{P}_{\widetilde{x}_{p}}(f), \breve{I}_{\widetilde{x}_{p}}(f)\right.$, $\left.N_{\tilde{x}_{p}}(f)\right\rangle(p=1,2)$ in $X$. Then, the comparison scheme is defined as follows:

(1) $S_{c}\left(\breve{S}_{\widetilde{x}}^{1}\right)<S_{c}\left(\breve{S}_{\widetilde{x}}^{2}\right)$, then $\breve{S}_{\widetilde{x}_{1}}<\breve{S}_{\widetilde{x}_{2}}$

(2) $S_{c}\left(\breve{S}_{\widetilde{x}}^{1}\right)>S_{c}\left(\breve{S}_{\widetilde{x}_{2}}\right)$, then $\breve{S}_{\widetilde{x}_{1}}>\breve{S}_{\widetilde{x}_{2}}$

(3) $S_{c}\left(\breve{S}_{\tilde{x}}^{1}\right)=S_{c}\left(\breve{S}_{\widetilde{x}}^{\sim}\right)$, then
(a) $A\left(\breve{S}_{\widetilde{x}_{1}}\right)<A\left(\breve{S}_{\widetilde{x}_{2}}\right)$, then $\breve{S}_{\widetilde{x}_{1}}<\breve{S}_{\widetilde{x}_{2}}$
(b) $A\left(\widetilde{S}_{\widetilde{x}_{1}}\right)>A\left(\widetilde{S}_{\widetilde{x}_{2}}\right)$, then $\widetilde{S}_{\widetilde{x}_{1}}>S_{\widetilde{x}_{2}}$
(c) $A\left(\widetilde{S}_{\widetilde{x}_{1}}\right)=A\left(\widetilde{S}_{\widetilde{x}_{2}}\right)$, then $\widetilde{S}_{\widetilde{x}_{1}} \approx \widetilde{S}_{\widetilde{x}_{2}}$

Definition 11. Let $\breve{S}_{\widetilde{x}_{p}}=\left\{x,\left\langle\left[\widehat{u}_{p}^{-}, \widehat{u}_{p}^{+}\right], \widehat{\alpha}_{p}\right\rangle,\left\langle\left[\widehat{v}_{p}^{-}, \widehat{v}_{p}^{+}\right], \widehat{\beta}_{p}\right\rangle\right.$, $\left.\left\langle\left[\widehat{w}_{p}^{-}, \widehat{w}_{p}^{+}\right], \widehat{\theta}_{p}\right\rangle\right\}$ be the collection of SCFNs where $(p=$ $1,2,3, \ldots, n)$ in $X$. The spherical cubic fuzzy weighted averaging (SCFWA) operator is illustrated as

$$
\operatorname{SCFWA}\left(\breve{S}_{\widetilde{x}_{1}}, \breve{S}_{\widetilde{x}_{2}}, \ldots, \breve{S}_{\widetilde{x}_{n}}\right)=\sum_{p=1}^{n} \gamma_{p} \breve{S}_{\widetilde{x}}^{P} \text {, }
$$

$\gamma_{p}(p=1,2,3, \ldots, n)$ are weight vectors with $\gamma_{p} \geq 0$ and $\sum_{p=1}^{n} \gamma_{p}=1$.

Definition 12. Let $\breve{S}_{\widetilde{x}_{p}}=\left\{x,\left\langle\left[\widehat{u}_{p}^{-}, \widehat{u}_{p}^{+}\right], \widehat{\alpha}_{p}\right\rangle,\left\langle\left[\widehat{v}_{p}^{-}, \widehat{v}_{p}^{+}\right], \widehat{\beta}_{p}\right\rangle\right.$, $\left.\left\langle\left[\widehat{w}_{p}^{-}, \widehat{w}_{p}^{+}\right], \widehat{\theta}_{p}\right\rangle\right\}$ be the collection of SCFNs where $(p=$ $1,2,3, \ldots, n)$ in $X$. The spherical cubic fuzzy ordered weighted average (SCFOWA) operator is defined as

$$
\operatorname{SCFOWA}\left(\breve{S}_{\widetilde{x}_{1}}, \breve{S}_{\widetilde{x}_{2}}, \ldots, \breve{S}_{\widetilde{x}_{n}}\right)=\sum_{p=1}^{n} \gamma_{p} \breve{S}_{\widetilde{x}_{\eta_{(p)}}},
$$

where $\gamma_{p}(p=1,2,3, \ldots, n)$ are weight vectors with $\gamma_{p} \in[0,1], \sum_{p=1}^{n} \gamma_{p}=1$, and the $p^{\text {th }}$ largest value is $\widetilde{S}_{\widetilde{x}_{\eta(p)}}$; therefore, the order is $\breve{S}_{\widetilde{x}_{\eta_{(1)}}}, \breve{S}_{\widetilde{x}_{\eta_{(2)}}}, \ldots, S_{\widetilde{x}_{\eta_{(}(n)}}$.

Definition 13. Let $\breve{S}_{\widetilde{x}_{p}}=\left\{x,\left\langle\left[\widehat{u}_{p}^{-}, \widehat{u}_{p}^{+}\right], \widehat{\alpha}_{p}\right\rangle,\left\langle\left[\widehat{v}_{p}^{-}, \widehat{v}_{p}^{+}\right], \widehat{\beta}_{p}\right\rangle\right.$, $\left.\left\langle\left[\widehat{w}_{p}^{-}, \widehat{w}_{p}^{+}\right], \widehat{\theta}_{p}\right\rangle\right\}$ be the collection of SCFNs where $(p=$ $1,2,3, \ldots, n)$ in $X$. The spherical cubic fuzzy hybrid weighted averaging (SCFHWA) operator is defined as follows:

$$
\operatorname{SCFWHA}\left(\breve{S}_{\widetilde{x}_{1}}, \breve{S}_{\widetilde{x}_{2}}, \ldots, \breve{S}_{\widetilde{x}_{n}}\right)=\sum_{p=1}^{n} \gamma_{p} \breve{S}_{\widetilde{x}_{\eta_{(p)}}}^{*}
$$

Here, the weighted vector is represented as $\gamma_{p}(p=1,2$, $\ldots \ldots, n)$ with $\gamma_{p} \geq 0$ and $\sum_{p=1}^{n} \psi_{p}=1$, and the $p^{\text {th }}$ largest weight value is $S_{\mathcal{*}_{*} \tilde{x}_{(p)}}\left(S_{\widetilde{x}_{\eta_{(p)}}}=\eta_{\mathbb{*}} S_{\widetilde{x}_{\eta_{(p)}}}\right)$, and the order is defined as $S_{\widetilde{x}_{\eta_{(1)}}} \geq S_{\widetilde{x}_{\eta_{(1)}}}^{*} \geq \ldots \geq S_{\widetilde{x}_{\eta_{(n)}}}$, and $w^{*}=\left(w_{1}^{*}, w_{2}^{*}, \ldots\right.$, 
$\left.w_{n}^{*}\right)$, and $\sum_{p=1}^{n} w_{p}^{*}=1$ with $w_{p}^{*} \geq 0$ representing the weighted vector.

Definition 14. Let $\breve{S}_{\widetilde{x}_{p}}=\left\{x,\left\langle\left[\widehat{u}_{p}^{-}, \widehat{u}_{p}^{+}\right], \widehat{\alpha}_{p}\right\rangle,\left\langle\left[\widehat{v}_{p}^{-}, \widehat{v}_{p}^{+}\right], \widehat{\beta}_{p}\right\rangle\right.$, $\left.\left\langle\left[\widehat{w}_{p}^{-}, \widehat{w}_{p}^{+}\right], \widehat{\theta}_{p}\right\rangle\right\}$ be the collection of SCFNs where $(p=$ $1,2,3, \ldots, n)$ in $X$. The spherical cubic fuzzy weighted geometric (SCFWG) operator is

$$
\operatorname{SCFWG}\left(\breve{S}_{\widetilde{x}_{1}}, \breve{S}_{\widetilde{x}_{2}}, \ldots, \breve{S}_{\widetilde{x}_{n}}\right)=\prod_{p=1}^{n}\left(\breve{S}_{\widetilde{x}_{p}}\right)^{\gamma_{p}},
$$

where the weight vector is represented $\gamma_{p}(p=1,2, \ldots, n)$ with $\gamma_{p} \geq 0$.

Definition 15. Let $\breve{S}_{\widetilde{x}_{p}}=\left\{x,\left\langle\left[\widehat{u}_{p}^{-}, \widehat{u}_{p}^{+}\right], \widehat{\alpha}_{p}\right\rangle,\left\langle\left[\widehat{v}_{p}^{-}, \widehat{v}_{p}^{+}\right], \widehat{\beta}_{p}\right\rangle\right.$, $\left.\left\langle\left[\widehat{w}_{p}^{-}, \widehat{w}_{p}^{+}\right], \widehat{\theta}_{p}\right\rangle\right\}$ be the collection of SCFNs where $(p=1,2,3, \ldots, n)$ in $X$. The spherical cubic fuzzy ordered weighted geometric (SCFOWG) operator is

$$
\operatorname{SCFOWG}\left(\breve{S}_{\widetilde{x}_{1}} \breve{S}_{\widetilde{x}_{2}}, \ldots, \breve{S}_{\widetilde{x}_{n}}\right)=\prod_{p=1}^{n}\left(\breve{S}_{\widetilde{x}_{\eta_{(p)}}}\right)^{\gamma_{p}} \text {, }
$$

and the $p^{\text {th }}$ largest weight value is $\breve{S}_{\widetilde{x} \eta_{(p)}}\left(\breve{S}_{\widetilde{x}_{\eta_{(p)}}}=\eta \breve{S}_{\widetilde{x} \eta_{(p)}}\right)$, and the order is defined as $\breve{S}_{\widetilde{x} \eta_{(1)}} \geq \breve{S}_{\widetilde{x} \eta_{(2)}}, \ldots, \geq \widetilde{S}_{\widetilde{x} \eta_{(n)}}$.

Definition 16. Let $\breve{S}_{\widetilde{x}_{p}}=\left\{x,\left\langle\left[\widehat{u}_{p}^{-}, \widehat{u}_{p}^{+}\right], \widehat{\alpha}_{p}\right\rangle,\left\langle\left[\widehat{v}_{p}^{-}, \widehat{v}_{p}^{+}\right], \widehat{\beta}_{p}\right\rangle\right.$, $\left.\left\langle\left[\widehat{w}_{p}^{-}, \widehat{w}_{p}^{+}\right], \widehat{\theta}_{p}\right\rangle\right\}$ be the collection of SCFNs where $(p=$ $1,2,3, \ldots, n)$ in $X$. The spherical cubic fuzzy hybrid weighted geometric (SCFHWG) operator is

$$
\operatorname{SCFHWG}\left(\breve{S}_{\widetilde{x}_{1}}, \breve{S}_{\widetilde{x}_{2}}, \ldots, \breve{S}_{\widetilde{x}_{n}}\right)=\prod_{p=1}^{n}\left(\breve{S}_{\widetilde{x}_{p}}\right)^{\gamma_{p}},
$$

where $\gamma_{p} \geq 0$ represent the weight vector where $(p=1,2,3$, $\ldots, n)$, and the $p^{\text {th }}$ largest weight value is $\breve{S}_{\widetilde{x}_{\eta(p)}^{*}}\left(\breve{S}_{\tilde{x} \eta_{(p)}^{*}}^{*}=\eta\right.$ $\breve{S}_{\left.\widetilde{x} \eta_{(p)}\right)}^{*}$, and the order is defined as $\breve{S}_{\widetilde{x} \eta_{(1)}}^{*} \geq \breve{S}_{\widetilde{x} \eta_{(1)}}^{*} \geq \ldots .$. $\geq \breve{S}_{\tilde{x} \eta_{(n)}^{*}}$. Also, associated weights are $\stackrel{*}{w}=\left(w_{1}^{*}, w_{2}, \ldots, w_{n}^{*}\right)$ with $\sum_{p=1}^{n} \stackrel{*}{w}_{p}=1$ and $\stackrel{*}{w}_{p} \geq 0$.

Definition 17. Assume that $(a, b) \in(0,1) \times(0,1)$ be real numbers and $r \geq 1$. Then, the norms of the Dombi are determined as

$$
\begin{aligned}
\widehat{T}(a, b)) & =\frac{1}{1+\left\{(1-a / a)^{r}+(1-b / b)^{r}\right\}^{1 / r}}, \\
\widehat{S}(a, b) & =1-\frac{1}{1+\left\{(1-a / a)^{r}+(1-b / b)^{r}\right\}^{1 / r}} .
\end{aligned}
$$

Example 1. Assume $a=0.4, b=0.5$, and $r=5$. Then,

$$
\begin{aligned}
& \widehat{T}(a, b)=\frac{1}{1+\left\{(1-0.4 / 0.4)^{5}+(1-0.7 / 0.7)^{5}\right\}^{1 / 5}}, \\
& \widehat{S}(a, b)=1-\frac{1}{1+\left\{(1-0.4 / 0.4)^{5}+(1-0.7 / 0.7)^{5}\right\}^{1 / 5}} .
\end{aligned}
$$

\section{Spherical Cubic Fuzzy Dombi Aggregated Operators}

Presently, we proposed the concept of spherical cubic fuzzy Dombi aggregated operations and discussed some of their characteristics in this section on the basis of Definition 7. We will introduce the spherical cubic fuzzy Dombi weighted averaging (SCFDWA) operator, spherical cubic fuzzy Dombi ordered weighted averaging (SCFDOWA) operator, spherical cubic fuzzy Dombi weighted geometric (SCFDWG) operator, spherical cubic fuzzy Dombi ordered weighted geometric (SCFDOWG) operator, and spherical cubic fuzzy Dombi hybrid weighted geometric (SCFDHWG) operator and studied its fundamental properties, i.e., boundary property, idempotency property, and monotonic property.

(1) $\breve{S}_{\widetilde{x}_{1}} \oplus_{D} \breve{S}_{\widetilde{x}_{2}}=$

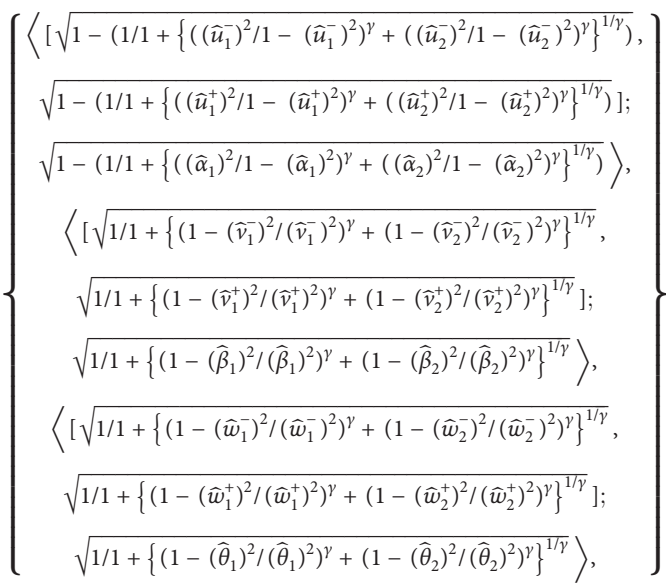

(2) $\breve{S}_{\widetilde{x}_{1}} \otimes_{D} \breve{S}_{\widetilde{x}_{2}}=$

$$
\begin{aligned}
& \left\langle\left[\sqrt{1 / 1+\left\{\left(1-\left(\widehat{u}_{1}^{-}\right)^{2} /\left(\widehat{u}_{1}^{-}\right)^{2}\right)^{\gamma}+\left(1-\left(\widehat{u}_{2}^{-}\right)^{2} /\left(\widehat{u}_{2}^{-}\right)^{2}\right)^{\gamma}\right.}\right\}^{1 / \gamma},\right. \\
& \left.\sqrt{1 / 1+\left\{\left(1-\left(\hat{u}_{1}^{+}\right)^{2} /\left(\widehat{u}_{1}^{+}\right)^{2}\right)^{\gamma}+\left(1-\left(\hat{u}_{2}^{+}\right)^{2} /\left(\widehat{u}_{2}^{+}\right)^{2}\right)^{\gamma}\right\}^{1 / \gamma}}\right] ; \\
& \left.\sqrt{1 / 1+\left\{\left(1-\left(\widehat{\alpha}_{1}\right)^{2} /\left(\widehat{\alpha}_{1}\right)^{2}\right)^{\gamma}+\left(1-\left(\widehat{\alpha}_{2}\right)^{2} /\left(\widehat{\alpha}_{2}\right)^{2}\right)^{\gamma}\right\}^{1 / \gamma}}\right\rangle, \\
& \left\langle\left[\sqrt{1-\left(1 / 1+\left\{\left(\left(\widehat{v}_{1}^{-}\right)^{2} / 1-\left(\widehat{v}_{1}^{-}\right)^{2}\right)^{\gamma}+\left(\left(\widehat{v}_{2}^{-}\right)^{2} / 1-\left(\widehat{v}_{2}^{-}\right)^{2}\right)^{\gamma}\right\}^{1 / \gamma}\right.}\right),\right. \\
& \left.\sqrt{1-\left(1 / 1+\left\{\left(\left(_{1}^{+}\right)^{2} / 1-\left(\hat{v}_{1}^{+}\right)^{2}\right)^{\gamma}+\left(\left(\hat{v}_{2}^{+}\right)^{2} / 1-\left(\hat{v}_{2}^{+}\right)^{2}\right)^{\gamma}\right\}^{1 / \gamma}\right)}\right] ; \\
& \left.\sqrt{1-\left(1 / 1+\left\{\left(\left(\hat{\beta}_{1}\right)^{2} / 1-\left(\hat{\beta}_{1}\right)^{2}\right)^{\gamma}+\left(\left(\widehat{\beta}_{2}\right)^{2} / 1-\left(\hat{\beta}_{2}\right)^{2}\right)^{\gamma}\right\}^{1 / \gamma}\right)}\right\rangle, \\
& \left\langle\left[\sqrt{1-\left(1 / 1+\left\{\left(\widehat{w}_{1}^{-}\right)^{2} / 1-\left(\widehat{w}_{1}^{-}\right)^{2}\right)^{\gamma}+\left(\left(\widehat{w}_{2}^{-}\right)^{2} / 1-\left(\widehat{w}_{2}^{-}\right)^{2}\right)^{\gamma}\right\}^{1 / \gamma}}\right),\right. \\
& \left.\sqrt{1-\left(1 / 1+\left\{\left(\left(\hat{w}_{1}^{+}\right)^{2} / 1-\left(\hat{w}_{1}^{+}\right)^{2}\right)^{\gamma}+\left(\left(\hat{w}_{2}^{+}\right)^{2} / 1-\left(\hat{w}_{2}^{+}\right)^{2}\right)^{\gamma}\right\}^{1 / \gamma}\right)}\right] ; \\
& \left.\sqrt{1-\left(1 / 1+\left\{\left(\left(\hat{\theta}_{1}\right)^{2} / 1-\left(\hat{\theta}_{1}\right)^{2}\right)^{\gamma}+\left(\left(\hat{\theta}_{2}\right)^{2} / 1-\left(\hat{\theta}_{2}\right)^{2}\right)^{1 / 1 / \gamma}\right)\right.}\right\rangle,
\end{aligned}
$$


(3) $\rho \cdot{ }_{D} \breve{S}_{\widetilde{x}_{1}}=\left\{\begin{array}{c}\left\langle\left[\sqrt{1-\left(1 / 1+\left\{\rho\left(\left(\hat{u}_{1}^{-}\right)^{2} / 1-\left(\widehat{u}_{1}^{-}\right)^{2}\right)^{2 \gamma}\right\}^{1 / \gamma}\right.}\right),\right. \\ \left.\left.\sqrt{1-\left(1 / 1+\left\{\rho\left(\left(\widehat{u}_{1}^{+}\right)^{2} / 1-\left(\widehat{u}_{1}^{+}\right)^{2}\right)^{2 \gamma}\right\}^{1 / \gamma}\right.}\right)\right] ; \\ \left.\left.\sqrt{1-\left(1 / 1+\left\{\rho\left(\left(\widehat{\alpha}_{1}\right)^{2} / 1-\left(\widehat{\alpha}_{1}\right)^{2}\right)^{2 \gamma}\right\}^{1 / \gamma}\right.}\right)\right\rangle, \\ \left\langle\left[\sqrt{1 / 1+\left\{\rho\left(1-\left(\widehat{v}_{1}^{-}\right)^{2} /\left(\widehat{v}_{1}^{-}\right)^{2}\right)^{2 \gamma}\right\}^{1 / \gamma}},\right.\right. \\ \left.\sqrt{1 / 1+\left\{\rho\left(1-\left(\hat{v}_{1}^{+}\right)^{2} /\left(\widehat{v}_{1}^{+}\right)^{2}\right)^{2 \gamma}\right\}^{1 / \gamma}}\right] ; \\ \left.\sqrt{1 / 1+\left\{\rho\left(1-\left(\widehat{\beta}_{1}\right)^{2} /\left(\widehat{\beta}_{1}\right)^{2}\right)^{2 \gamma}\right\}^{1 / \gamma}}\right\rangle, \\ \left\langle\sqrt{1 / 1+\left\{\rho\left(1-\left(\widehat{w}_{1}^{-}\right)^{2} /\left(\widehat{w}_{1}^{-}\right)^{2}\right)^{2 \gamma}\right\}^{1 / \gamma}},\right. \\ \left.\sqrt{1 / 1+\left\{\rho\left(1-\left(\widehat{w}_{1}^{+}\right)^{2} /\left(\widehat{w}_{1}^{+}\right)^{2}\right)^{2 \gamma}\right\}^{1 / \gamma}}\right] ; \\ \left.\sqrt{1 / 1+\left\{\rho\left(1-\left(\widehat{\theta}_{1}\right)^{2} /\left(\widehat{\theta}_{1}\right)^{2}\right)^{\gamma}\right\}^{1 / \gamma}}\right\rangle,\end{array}\right\}$

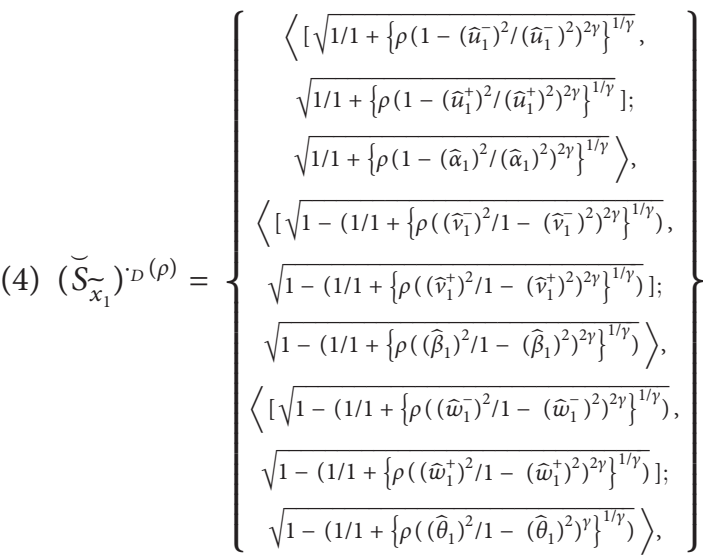

Definition 18. Let two SCFNs be $\breve{S}_{\widetilde{x}_{1}}=\left\{x,\left\langle\left[\widehat{u}_{1}^{-}, \widehat{u}_{1}^{+}\right], \widehat{\alpha}_{1}\right\rangle,\left\langle\left[\widehat{v}_{1}^{-}, \widehat{v}_{1}^{+}\right], \widehat{\beta}_{1}\right\rangle,\left\langle\left[\widehat{w}_{1}^{-}, \widehat{w}_{1}^{+}\right], \widehat{\theta}_{1}\right\rangle\right\} \quad$ and $\breve{S}_{\widetilde{x}_{2}}=\left\{x,\left\langle\left[\widehat{u}_{2}^{-}, \widehat{u}_{2}^{+}\right], \widehat{\alpha}_{2}\right\rangle,\left\langle\left[\widehat{v}_{2}^{-}, \widehat{v}_{2}^{+}\right], \widehat{\beta}_{2}\right\rangle,\left\langle\left[\widehat{w}_{2}^{-}, \widehat{w}_{2}^{+}\right], \widehat{\theta}_{2}\right\rangle\right\}$ in $X$ and $\gamma \geq 0$. Then, the operations of SCFNs on the basis of Dombi operation are presented as

4.1. Spherical Cubic Dombi Weighted Averaging Operations. In the view of characterized Dombi operations of SCFNs, we describe the following weighted averaging aggregated operators.

Definition 19. For any collection of SCFNs, $\breve{S}_{\widetilde{x}_{p}}=\{x$, $\left.\left\langle\left[\widehat{u}_{p}^{-}, \widehat{u}_{p}^{+}\right], \quad \widehat{\alpha}_{p}\right\rangle,\left\langle\left[\widehat{v}_{p}^{-}, \widehat{v}_{p}^{+}\right], \widehat{\beta}_{p}\right\rangle,\left\langle\left[\widehat{w}_{p}^{-}, \widehat{w}_{p}^{+}\right], \widehat{\theta}_{p}\right\rangle\right\}(p=1,2$, $\ldots, n)$ in $X$. Then, structure of spherical cubic fuzzy Dombi weighted averaging (SCFDWA) operator is

$$
\operatorname{SCFDWA}\left(\breve{S}_{\widetilde{x}_{1}}, \breve{S}_{\widetilde{x}_{2}}, \ldots, \breve{S}_{\widetilde{x}_{n}}\right)=\sum_{p=1}^{n} \gamma_{p} \breve{S}_{\widetilde{x}_{p}},
$$

where $\gamma_{p}$ is the weight vector with $(p=1,2, \ldots, n)$ and $\sum_{p=1}^{n} \gamma_{p}=1$.

Theorem 1. For any collections of SCFNs, $\breve{S}_{\widetilde{x}_{p}}=\left\{x,\left\langle\left[\widehat{u}_{p}^{-}\right.\right.\right.$, $\left.\left.\left.\widehat{u}_{p}^{+}\right], \widehat{\alpha}_{p}\right\rangle,\left\langle\left[\widehat{v}_{p}^{-}, \widehat{v}_{p}^{+}\right], \widehat{\beta}_{p}\right\rangle,\left\langle\left[\widehat{w}_{p}^{-}, \widehat{w}_{p}^{+}\right], \widehat{\theta}_{p}\right\rangle\right\}(p=1,2, \ldots, n)$ in $X$. Then, the spherical cubic Dombi weighted averaging (SCFDWA) operator is defined using the operations on Dombi with some positive constant $\varepsilon>0$ as follows:

$$
\begin{aligned}
& \operatorname{SCFDWA}\left(\breve{S}_{\widetilde{x}_{1}}, \breve{S}_{\widetilde{x}_{2}}, \ldots, \breve{S}_{\widetilde{x}_{n}}\right)=
\end{aligned}
$$

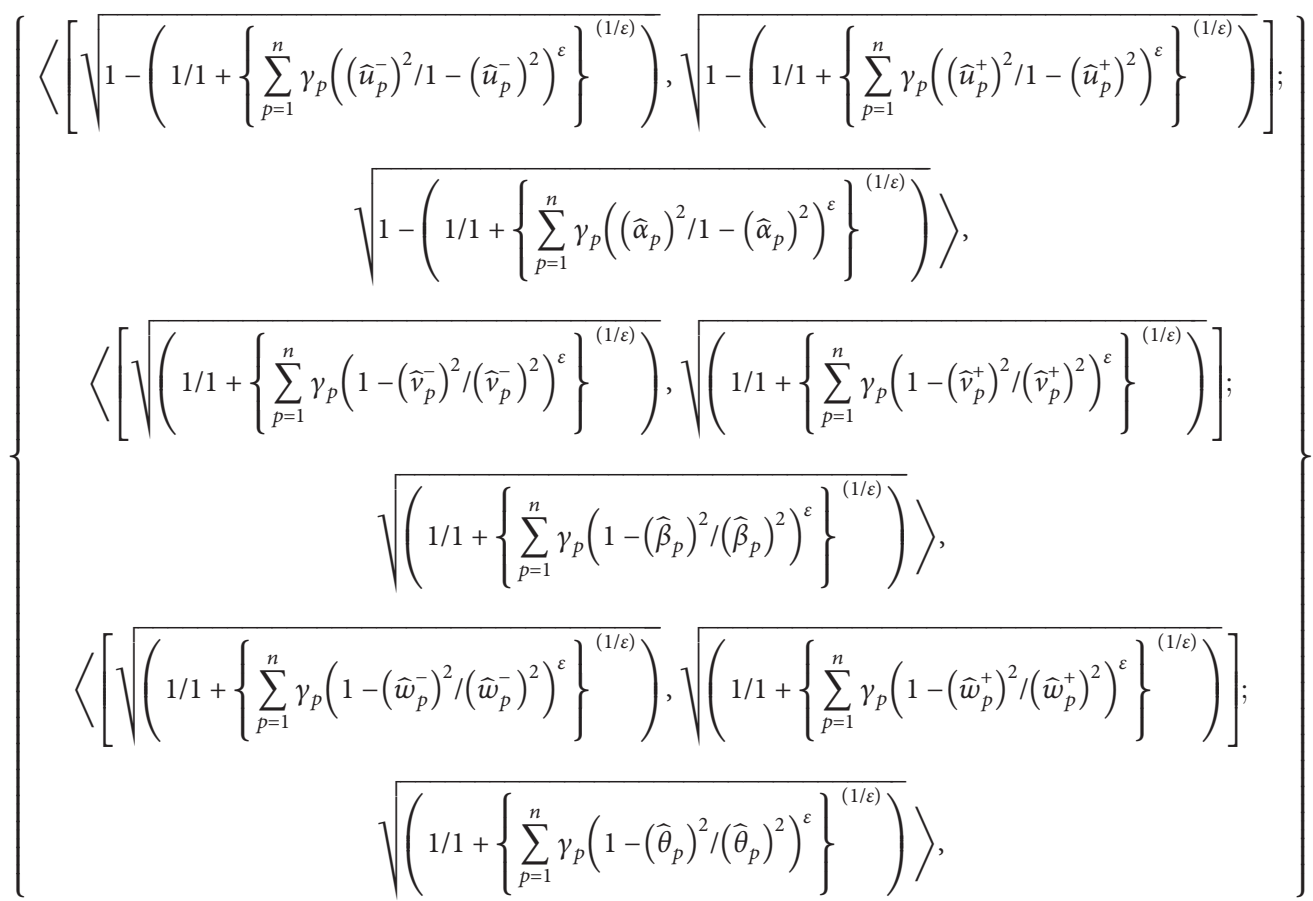


where $\gamma_{p}$ represents the weight vectors $\gamma_{p} \geq 0$ with $(p=1,2, \ldots, n)$ and $\sum_{p=1}^{n} \gamma_{p}=1$.
Proof. We will prove it by mathematical induction, so Theorem 1 is true for $n=2$.

$$
\begin{aligned}
& \operatorname{SCFDWA}\left(\breve{S}_{\widetilde{x}_{1}}+\breve{S}_{\widetilde{x}_{2}}\right)=\gamma_{1} \breve{S}_{\widetilde{x}_{1}}+\gamma_{2} \breve{S}_{\widetilde{x}_{2}} \\
& \left\langle\left[\sqrt{1-\left(\frac{1}{1+\left\{\gamma_{1}\left(\left(\widehat{u}_{1}^{-}\right)^{2} / 1-\left(\widehat{u}_{1}^{-}\right)^{2}\right)^{\varepsilon}+\gamma_{2}\left(\left(\widehat{u}_{2}^{-}\right)^{2} / 1-\left(\widehat{u}_{2}^{-}\right)^{2}\right)^{\varepsilon}\right\}^{1 / \varepsilon}}\right)},\right.\right. \\
& \left.\sqrt{1-\left(\frac{1}{1+\left\{\gamma_{1}\left(\left(\widehat{u}_{1}^{+}\right)^{2} / 1-\left(\widehat{u}_{1}^{+}\right)^{2}\right)^{\varepsilon}+\gamma_{2}\left(\left(\widehat{u}_{2}^{+}\right)^{2} / 1-\left(\widehat{u}_{2}^{+}\right)^{2}\right)^{\varepsilon}\right\}^{1 / \varepsilon}}\right)}\right] \\
& \sqrt{1-\left(\frac{1}{1+\left\{\gamma_{1}\left(\left(\widehat{\alpha}_{1}\right)^{2} / 1-\left(\widehat{\alpha}_{1}\right)^{2}\right)^{\varepsilon}+\gamma_{2}\left(\left(\widehat{\alpha}_{2}\right)^{2} / 1-\left(\widehat{\alpha}_{2}\right)^{2}\right)^{\varepsilon}\right\}^{1 / \varepsilon}}\right)}, \\
& \left\langle\sqrt{\frac{1}{1+\left\{\gamma_{1}\left(1-\left(\widehat{v}_{1}^{-}\right)^{2} /\left(\widehat{v}_{1}^{-}\right)^{2}\right)^{\varepsilon}+\gamma_{2}\left(1-\left(\widehat{v}_{2}^{-}\right)^{2} /\left(\widehat{v}_{2}^{-}\right)^{2}\right)^{\varepsilon}\right\}^{1 / \varepsilon}}},\right. \\
& =\{ \\
& \left.\sqrt{\frac{1}{1+\left\{\gamma_{1}\left(1-\left(\widehat{v}_{1}^{+}\right)^{2} /\left(\widehat{v}_{1}^{+}\right)^{2}\right)^{\varepsilon}+\gamma_{2}\left(1-\left(\widehat{v}_{2}^{+}\right)^{2} /\left(\widehat{v}_{2}^{+}\right)^{2}\right)^{\varepsilon}\right\}^{1 / \varepsilon}}}\right] \\
& \left.\sqrt{\frac{1}{1+\left\{\gamma_{1}\left(1-\left(\widehat{\beta}_{1}\right)^{2} /\left(\widehat{\beta}_{1}\right)^{2}\right)^{\varepsilon}+\gamma_{2}\left(1-\left(\widehat{\beta}_{2}\right)^{2} /\left(\widehat{\beta}_{2}\right)^{2}\right)^{\varepsilon}\right\}^{1 / \varepsilon}}}\right\rangle, \\
& \left\langle\sqrt{\frac{1}{1+\left\{\gamma_{1}\left(1-\left(\widehat{w}_{1}^{-}\right)^{2} /\left(\widehat{w}_{1}^{-}\right)^{2}\right)^{\varepsilon}+\gamma_{2}\left(1-\left(\widehat{w}_{2}^{-}\right)^{2} /\left(\widehat{w}_{2}^{-}\right)^{2}\right)^{\varepsilon}\right\}^{1 / \varepsilon}}},\right. \\
& \left.\sqrt{\frac{1}{1+\left\{\gamma_{1}\left(1-\left(\widehat{w}_{1}^{+}\right)^{2} /\left(\widehat{w}_{1}^{+}\right)^{2}\right)^{\varepsilon}+\gamma_{2}\left(1-\left(\widehat{w}_{2}^{+}\right)^{2} /\left(\widehat{w}_{2}^{+}\right)^{2}\right)^{\varepsilon}\right\}^{1 / \varepsilon}}}\right] ;, \\
& \sqrt{\frac{1}{1+\left\{\gamma_{1}\left(1-\left(\hat{\theta}_{1}\right)^{2} /\left(\widehat{\theta}_{1}\right)^{2}\right)^{\varepsilon}+\gamma_{2}\left(1-\left(\hat{\theta}_{2}\right)^{2} /\left(\widehat{\theta}_{2}\right)^{2}\right)^{\varepsilon}\right\}^{1 / \varepsilon}}}
\end{aligned}
$$


Now, assume that equation (9) is true.

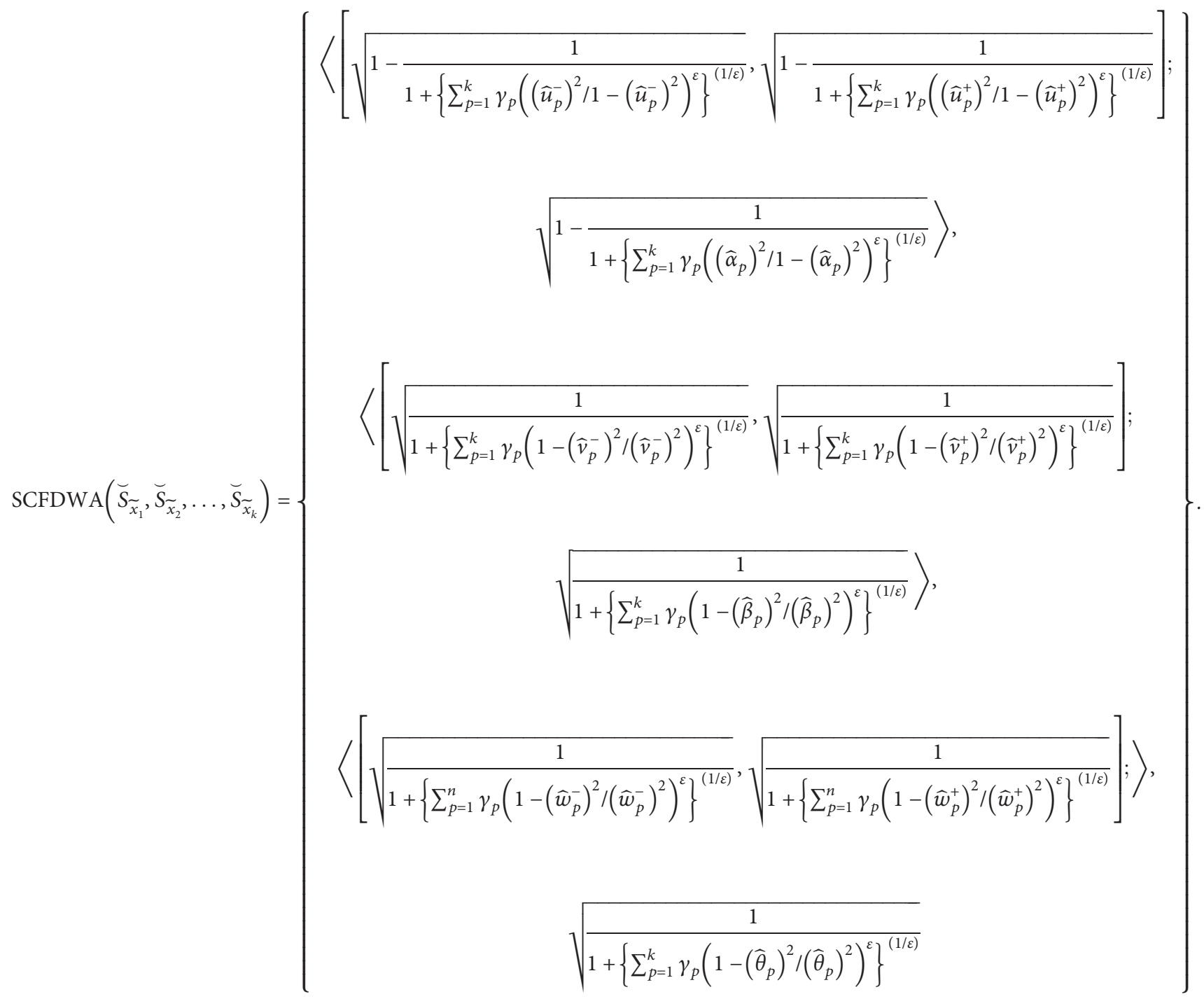

Now, we will prove equation (19) for $n=k+1$, i.e., 


$$
\begin{aligned}
& \operatorname{SCFDWA}\left(\breve{S}_{\widetilde{x}_{1}}, \breve{S}_{\widetilde{x}_{2}}, \ldots, \breve{S}_{\% \widetilde{x}_{k+1}}\right)=\sum_{p=1}^{k} \gamma_{k} S_{\widetilde{x}_{k}}+\gamma_{k+1} S_{\widetilde{x}_{k+1}},
\end{aligned}
$$

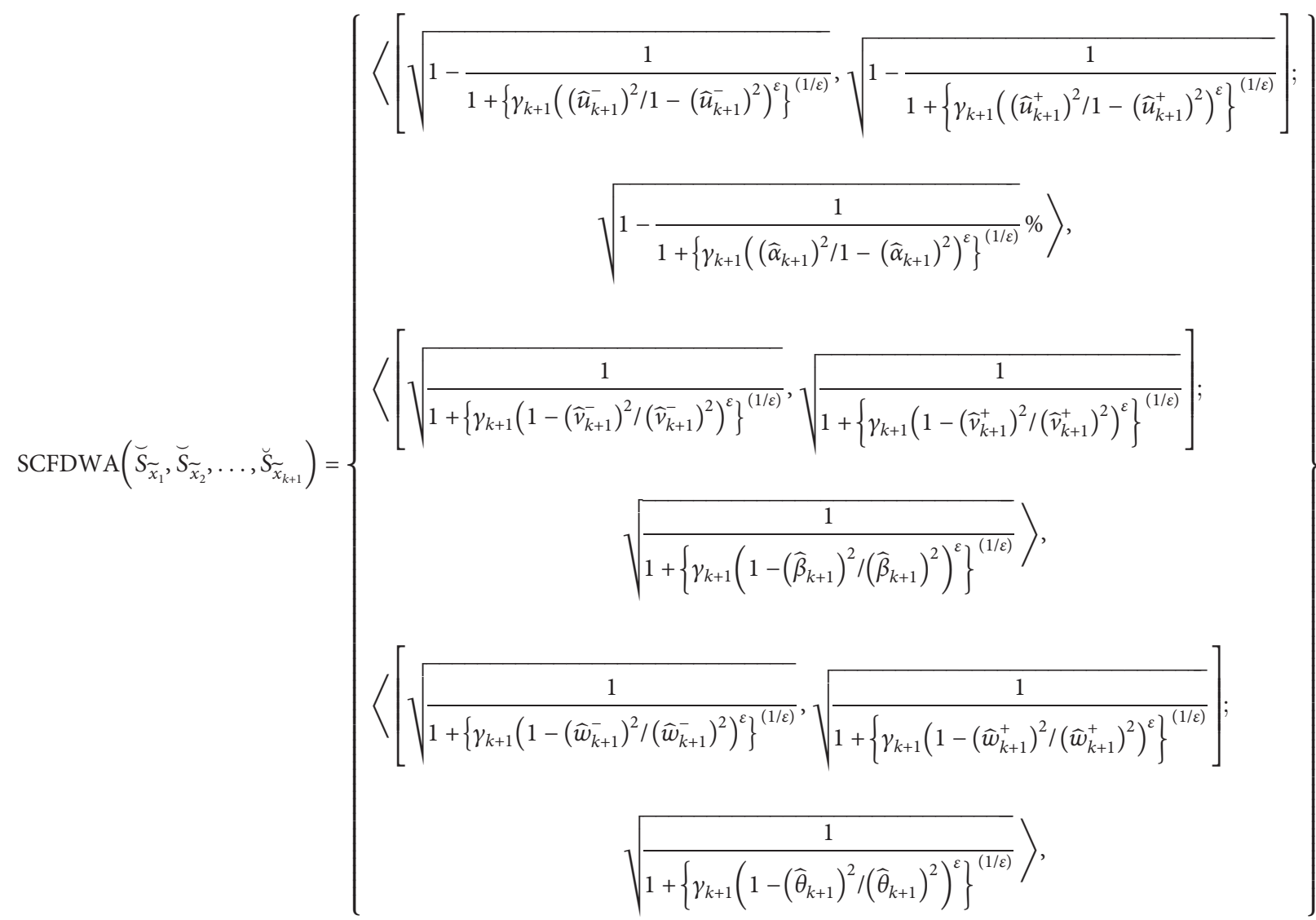

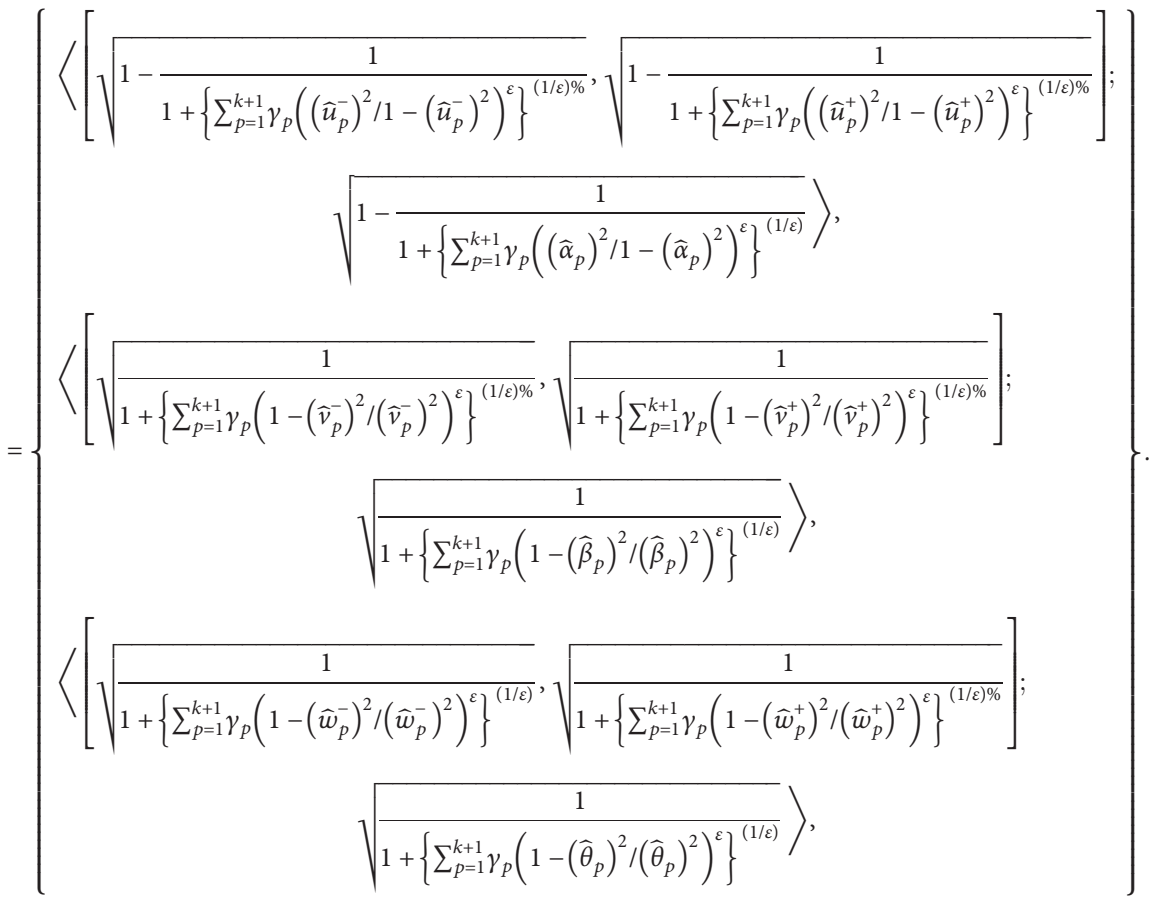

So, by the mathematical induction, it is true for all $n$. 


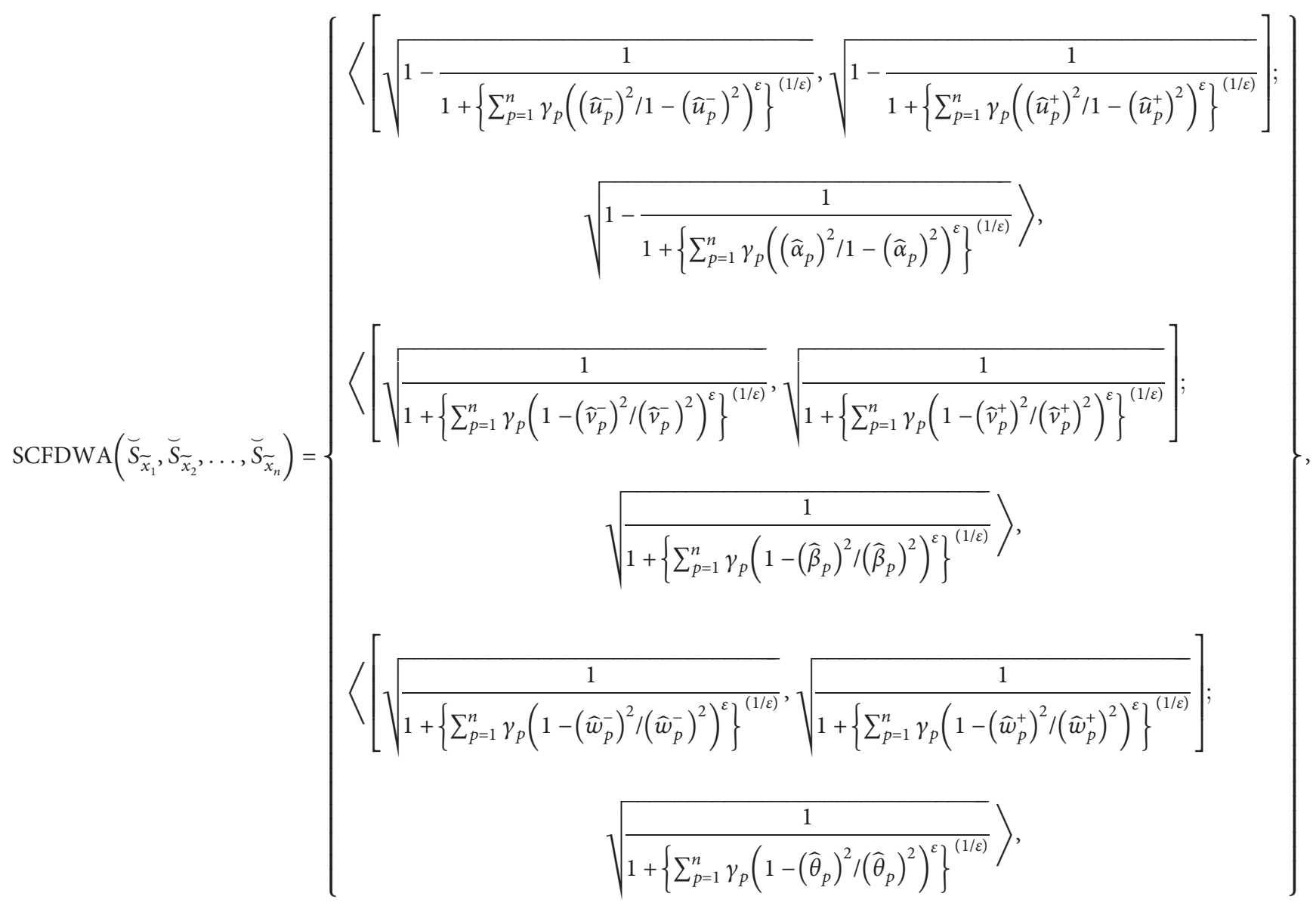

which we have to prove.

The properties of SCFDWA are as follows:

(1) Idempotency. We have the collection of SCFNs $\breve{S}_{\widetilde{x}_{p}}=$ $\left\{x,\left\langle\left[\widehat{u}_{p}^{-}, \widehat{u}_{p}^{+}\right], \widehat{\alpha}_{p}\right\rangle,\left\langle\left[\widehat{v}_{p}^{-}, \widehat{v}_{p}^{+}\right], \widehat{\beta}_{p}\right\rangle,\left\langle\left[\widehat{w}_{p}^{-}, \widehat{w}_{p}^{+}\right], \quad \widehat{\theta}_{p}^{x_{p}}\right\rangle\right\}$ $(p=1,2, \ldots, n)$ in $X$. Then, the collection of SCFNs $\breve{S}_{\widetilde{x}_{p}}(p=1,2, \ldots, n)$ is equal, i.e.,

$$
\operatorname{SCFDWA}\left(\breve{S}_{\widetilde{x}_{1}}, \breve{S}_{\widetilde{x}_{2}}, \ldots, \breve{S}_{\widetilde{x}_{n}}\right)=\breve{S}_{\widetilde{x}}
$$

(2) Boundary. We have the collection of SCFNs $\breve{S}_{\widetilde{x}_{p}}=$ $\left\{x,\left\langle\left[\widehat{u}_{p}^{-}, \widehat{u}_{p}^{+}\right], \widehat{\alpha}_{p}\right\rangle,\left\langle\left[\widehat{v}_{p}^{-}, \widehat{v}_{p}^{+}\right], \widehat{\beta}_{p}\right\rangle,\left\langle\left[\widehat{w}_{p}^{-}, \widehat{w}_{p}^{+}\right], \quad \widehat{\theta}_{p}^{p}\right\rangle\right\}$ $(p=1,2, \ldots, n)$ in $X$.

$$
\begin{array}{r}
\breve{S}_{\widetilde{x}_{p}}^{-}=\left\langle\left(\left[\min _{p} \widehat{u}_{p}^{-}, \min _{p} \widehat{u}_{p}^{+}\right], \min _{p} \widehat{\alpha}_{p}\right),\left(\left[\max _{p} \widehat{v}_{p}^{-}, \max _{p} \widehat{v}_{p}^{+}\right], \max _{p} \widehat{\beta}_{p}\right),\left(\left[\max _{p} \widehat{w}_{p}^{-}, \max _{p} \widehat{w}_{p}^{+}\right], \max _{p} \widehat{\theta}_{p}\right)\right\rangle, \\
\breve{S}_{\widetilde{x}_{p}}^{+}=\left\langle\left(\left[\max _{p} \widehat{u}_{p}^{-}, \max _{p} \widehat{u}_{p}^{+}\right], \max _{p} \widehat{\alpha}_{p}\right),\left(\left[\min _{p} \widehat{v}_{p}^{-}, \min _{p} \widehat{v}_{p}^{+}\right], \min _{p} \widehat{\beta}_{p}\right),\left(\left[\min _{p} \widehat{w}_{p}^{-}, \min _{p} \widehat{w}_{p}^{+}\right], \min _{p} \widehat{\theta}_{p}\right)\right\rangle . \\
\operatorname{SCFDWA}\left(\breve{S}_{\widetilde{x}_{1}}, \breve{S}_{\widetilde{x}_{2}}, \ldots, \breve{S}_{\widetilde{x}_{n}}\right) \subseteq \operatorname{SCFDWA}\left(\breve{S}_{\widetilde{x}_{1}}, \breve{S}_{\widetilde{x}_{2}}, \ldots, \breve{S}_{\widetilde{x}_{n}}\right) .
\end{array}
$$

Thus,

$$
\breve{S}_{\widetilde{x}_{p}}^{-} \leq \operatorname{SCFDWA}\left(\breve{S}_{\widetilde{x}_{1}}, \breve{S}_{\widetilde{x}_{2}}, \ldots, \breve{S}_{\widetilde{x}_{n}}\right) \leq \breve{S}_{\widetilde{x}_{p}}^{+} .
$$

(3) Monotonicity. We have the collection of SCFNs $\breve{S}_{\widetilde{x}_{p}}=$ $\left\{x,\left\langle\left[\widehat{u}_{p}^{-}, \widehat{u}_{p}^{+}\right], \widehat{\alpha}_{p}\right\rangle,\left\langle\left[\widehat{v}_{p}^{-}, \widehat{v}_{p}^{+}\right], \widehat{\beta}_{p}\right\rangle,\left\langle\left[\widehat{w}_{p}^{-}, \widehat{w}_{p}^{+}\right], \quad \widehat{\theta}_{p}^{p}\right\rangle\right\}$ $(p=1,2, \ldots, n)$ in $X . \breve{S}_{\widetilde{x}_{p}} \subseteq \breve{S}_{\widetilde{x}_{p}}^{k}$ for $(p=1,2, \ldots, n)$, then

Definition 20. We have the collection of SCFNs $\breve{S}_{\widetilde{x}_{p}}=\left\{x,\left\langle\left[\widehat{u}_{p}^{-}, \widehat{u}_{p}^{+}\right], \widehat{\alpha}_{p}\right\rangle, \quad\left\langle\left[\widehat{v}_{p}^{-}, \widehat{v}_{p}^{+}\right], \widehat{\beta}_{p}\right\rangle,\left\langle\left[\widehat{w}_{p}^{-}, \widehat{w}_{p}^{+}\right], \widehat{\theta}_{p}\right\rangle\right\}$ $(p=1,2, \ldots, n)$ in $X$. The spherical cubic fuzzy Dombi 
ordered weighted average (SCDOWA) operator is defined as

$$
\operatorname{SCFDWA}\left(\breve{S}_{\widetilde{x}_{1}}, \breve{S}_{\widetilde{x}_{2}}, \ldots, \breve{S}_{\widetilde{x}_{n}}\right)=\sum_{p=1}^{n} \gamma_{p} \breve{S}_{\widetilde{x}_{p}},
$$

where $\gamma_{p}(p=1,2,3, \ldots, n)$ represent the weight vectors satisfying $\sum_{p=1}^{n} \gamma_{p}=1$ and $\gamma_{p} \geq 0$, and the largest $p^{\text {th }}$ weighted value is $\breve{S}_{\widetilde{x}_{\eta(p)}}$, and the total order is $S_{\widetilde{x}_{\eta(1)}} \geq S_{\widetilde{x}_{\eta(2)}} \geq \ldots \geq S_{\widetilde{x}_{\eta(n)}} \cdot$

Theorem 2. We have the collection of SCFNs $\widehat{S}_{\widetilde{x}_{p}}=\left\{x,\left\langle\left[\widehat{u}_{p}^{-}, \widehat{u}_{p}^{+}\right], \widehat{\alpha}_{p}\right\rangle, \quad\left\langle\left[\widehat{v}_{p}^{-}, \widehat{v}_{p}^{+}\right], \widehat{\beta}_{p}\right\rangle,\left\langle\left[\widehat{w}_{p}^{-}, \widehat{w}_{p}^{+}\right], \widehat{\theta}_{p}\right\rangle\right.$ \}$(p=1,2, \ldots, n)$ in $X$. The spherical cubic fuzzy Dombi ordered weighted average (SCDOWA) operator is defined as $\varepsilon>0$.

$$
\begin{aligned}
& \operatorname{SCFDWA}\left(\breve{S}_{\widetilde{x}_{1}}, \breve{S}_{\widetilde{x}_{2}}, \ldots, \breve{S}_{\widetilde{x}_{n}}\right)=
\end{aligned}
$$

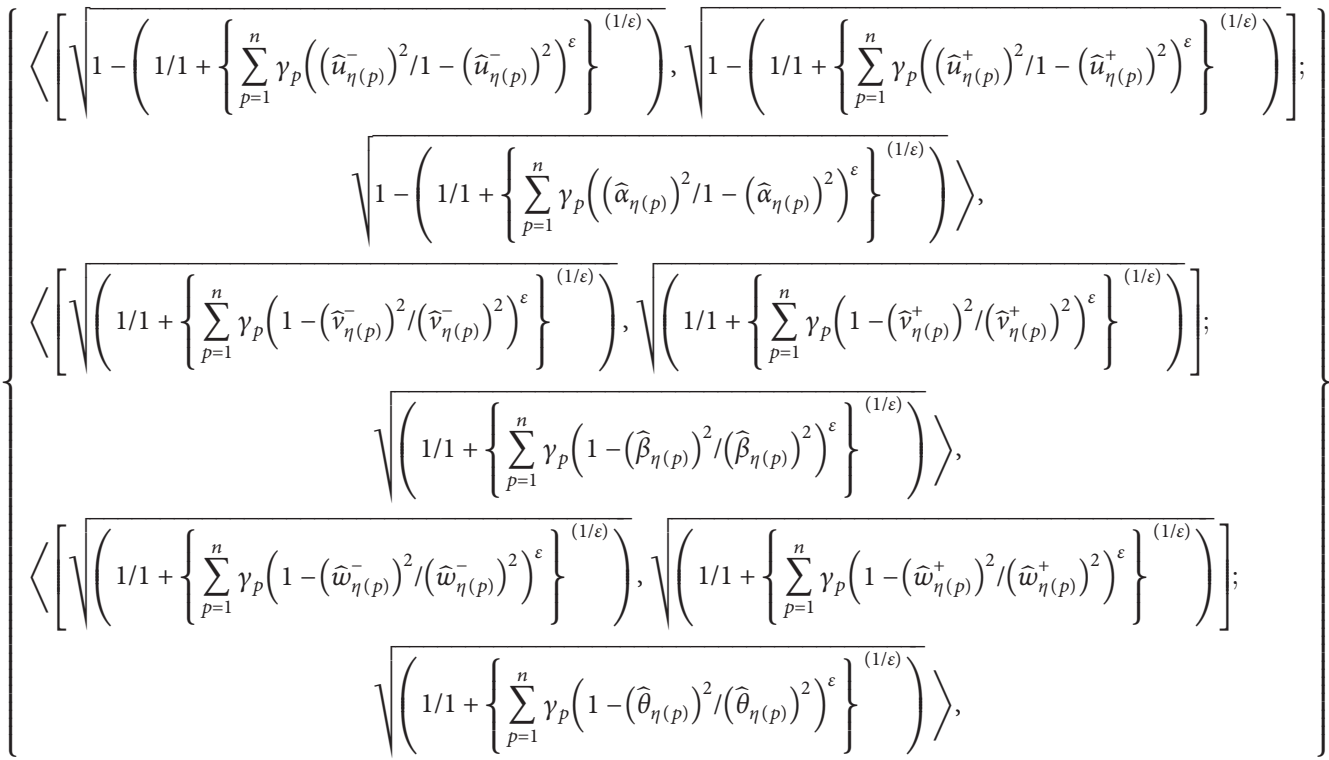

where $\gamma_{p}$ represent the weight vector where $(p=1,2,3, \ldots, n)$ and $\sum_{p=1}^{n} y_{p}=1$, and the largest weight is $S_{\widetilde{x}_{\eta(p)}}$ and the total order is $S_{\widetilde{x}_{\eta(1)}} \geq S_{\widetilde{x}_{\eta(2)}} \geq \ldots \geq S_{\widetilde{x}_{\eta(n)}}$.

Proof. The proof is similar to Theorem 1.

The properties of SCFDOWA are as follows:

(1) Idempotency. We have the collection of SCFNs $\breve{S}_{\widetilde{x}_{p}}=$ $\left\{x,\left\langle\left[\widehat{u}_{p}^{-}, \widehat{u}_{p}^{+}\right], \widehat{\alpha}_{p}\right\rangle\right.$, $\left.\left\langle\left[\widehat{v}_{p}^{-}, \widehat{v}_{p}^{+}\right], \widehat{\beta}_{p}\right\rangle,\left\langle\left[\widehat{w}_{p}^{-}, \widehat{w}_{p}^{+}\right], \widehat{\theta}_{p}\right\rangle\right\}(p=1,2, \ldots, n)$ in $X$. Then, we say collection of SCFNs $S_{\widetilde{x}_{p}}(p=1,2, \ldots, n)$ is equal. i.e.,

$$
\operatorname{SCFDOWA}\left(\breve{S}_{\widetilde{x}_{1}}, \breve{S}_{\widetilde{x}_{2}}, \ldots, \breve{S}_{\widetilde{x}_{n}}\right)=\breve{S}_{\widetilde{x}}
$$

(2) Boundary. We have the collection of SCFNs $\breve{S}_{\widetilde{x}}=$ $\left\{x,\left\langle\left[\widehat{u}_{p}^{-}, \widehat{u}_{p}^{+}\right], \widehat{\alpha}_{p}\right\rangle, \quad\left\langle\left[\widehat{v}_{p}^{-}, \widehat{v}_{p}^{+}\right], \widehat{\beta}_{p}\right\rangle,\left\langle\left[\widehat{w}_{p}^{-}, \widehat{w}_{p}^{+}\right], \widehat{\theta}_{p}^{p}\right\rangle\right\}$ $(p=1,2, \ldots, n)$ in $X$.

$$
\begin{aligned}
& \breve{S}_{\widetilde{x}_{p}}^{-}=\left\langle\left(\left[\min _{p} \widehat{u}_{p}^{-}, \min _{p} \widehat{u}_{p}^{+}\right], \min _{p} \widehat{\alpha}_{p}\right),\left(\left[\max _{p} \widehat{v}_{p}^{-}, \max _{p} \widehat{v}_{p}^{+}\right], \max _{p} \widehat{\beta}_{p}\right),\left(\left[\max _{p} \widehat{w}_{p}^{-}, \max _{p} \widehat{w}_{p}^{+}\right], \max _{p} \widehat{\theta}_{p}\right)\right\rangle, \\
& \breve{S}_{\widetilde{x}_{p}}^{+}=\left\langle\left(\left[\max _{p} \widehat{u}_{p}^{-}, \max _{p} \widehat{u}_{p}^{+}\right], \max _{p} \widehat{\alpha}_{p}\right),\left(\left[\min _{p} \widehat{v}_{p}^{-}, \min _{p} \widehat{v}_{p}^{+}\right], \min _{p} \widehat{\beta}_{p}\right),\left(\left[\min _{p} \widehat{w}_{p}^{-}, \min _{p} \widehat{w}_{p}^{+}\right], \min _{p} \widehat{\theta}_{p}\right)\right\rangle .
\end{aligned}
$$

Thus,

$$
\breve{S}_{\widetilde{x}_{p}}^{-} \leq \operatorname{SCFDOWA}\left(\breve{S}_{\widetilde{x}_{1}}, \breve{S}_{\widetilde{x}_{2}}, \ldots, \breve{S}_{\widetilde{x}_{n}}\right) \leq \breve{S}_{\widetilde{x}_{p}}^{+} .
$$

(3) Monotonicity. We have the collection of SCFNs $\widetilde{S}_{\widetilde{x}_{p}}=$ $\left\{x,\left\langle\left[\widehat{u}_{p}^{-}, \widehat{u}_{p}^{+}\right], \widehat{\alpha}_{p}\right\rangle, \quad\left\langle\left[\widehat{v}_{p}^{-}, \widehat{v}_{p}^{+}\right], \widehat{\beta}_{p}\right\rangle,\left\langle\left[\widehat{w}_{p}^{-}, \widehat{w}_{p}^{+}\right], \widehat{\theta}_{p}\right\rangle\right\}$ $(p=1,2, \ldots, n)$ in $X . \breve{S}_{\widetilde{x}_{p}} \subseteq \breve{S}_{\widetilde{x}_{p}}^{k}$ for $(p=1,2, \ldots, n)$, then

$$
\operatorname{SCFDOWA}\left(\breve{S}_{\widetilde{x}_{1}}, \breve{S}_{\widetilde{x}_{2}}, \ldots, \breve{S}_{\widetilde{x}_{n}}\right) \subseteq \operatorname{SCFDOWA}\left(\breve{S}_{\widetilde{x}_{1}}^{k}, \breve{S}_{\widetilde{x}_{2}}, \ldots, \breve{S}_{\widetilde{x}_{n}}^{k}\right) \text {. }
$$


Definition 21. We have the collection of SCFNs $\breve{S}_{\widetilde{x}_{p}}=\left\{x,\left\langle\left[\widehat{u}_{p}^{-}, \widehat{u}_{p}^{+}\right], \widehat{\alpha}_{p}\right\rangle, \quad\left\langle\left[\widehat{v}_{p}^{-}, \widehat{v}_{p}^{+}\right], \widehat{\beta}_{p}\right\rangle,\left\langle\left[\widehat{w}_{p}^{-}, \widehat{w}_{p}^{+}\right], \widehat{\theta}_{p}\right\rangle\right\}$ $(p=1,2, \ldots, n)$ in $X$. The spherical cubic fuzzy Dombi hybrid weighted average (SCFDHWA) operator is

$$
\operatorname{SCFDHWA}\left(\breve{S}_{\widetilde{x}_{1}}, \breve{S}_{\widetilde{x}_{2}}, \ldots, \breve{S}_{\widetilde{x}_{n}}\right)=\sum_{p=1}^{n} \gamma_{p} \breve{S}_{\widetilde{x}_{\eta(p)}}^{*},
$$

where $\gamma_{p}(p=1,2,3, \ldots, n)$ represent the weight vectors satisfying $\sum_{p=1}^{n} \gamma_{p}=1$ and $(p=1,2, \ldots, n)$, and $p^{\text {th }}$ largest value is $\breve{S}_{\widetilde{x}_{\eta(p)}}^{*}\left(\breve{S}_{\widetilde{x}_{\eta(p)}^{*}}^{*}=n \gamma_{p} \breve{S}_{\widetilde{x}_{\eta(p)}^{*}}^{*}, p \in N\right)$, and the total order is $\quad \breve{S}_{\widetilde{x}_{\eta(1)}}^{*} \geq \breve{S}_{\widetilde{x}_{\eta(2)}}^{*} \geq \ldots \geq \breve{S}_{\widetilde{x}_{\eta(n)}^{*}}^{*} \quad$ where $\quad w_{p}^{*} \geq 0, \sum_{p=1}^{n} w_{p}^{*}=$ $1 w_{p}^{*}=\left(w_{1}^{*}, w_{2}^{*}, \ldots, w_{n}^{*}\right)$.

Theorem 3. We have the collection of SCFNs $\breve{S}_{\widetilde{x}_{p}}=\left\{x,\left\langle\left[\widehat{u}_{p}^{-}, \widehat{u}_{p}^{+}\right], \widehat{\alpha}_{p}\right\rangle, \quad\left\langle\left[\widehat{v}_{p}^{-}, \widehat{v}_{p}^{+}\right], \widehat{\beta}_{p}\right\rangle,\left\langle\left[\widehat{w}_{p}^{-}, \widehat{w}_{p}^{+}\right], \widehat{\theta}_{p}\right\rangle\right\}$ $(p=1,2, \ldots, n)$ in $X$. The spherical cubic fuzzy Dombi hybrid weighted average (SCFDHWA) operator is defined as

$\operatorname{SCFDHWA}\left(\breve{S}_{\widetilde{x}_{1}}, \breve{S}_{\widetilde{x}_{2}}, \ldots, \breve{S}_{\widetilde{x}_{n}}\right)=$

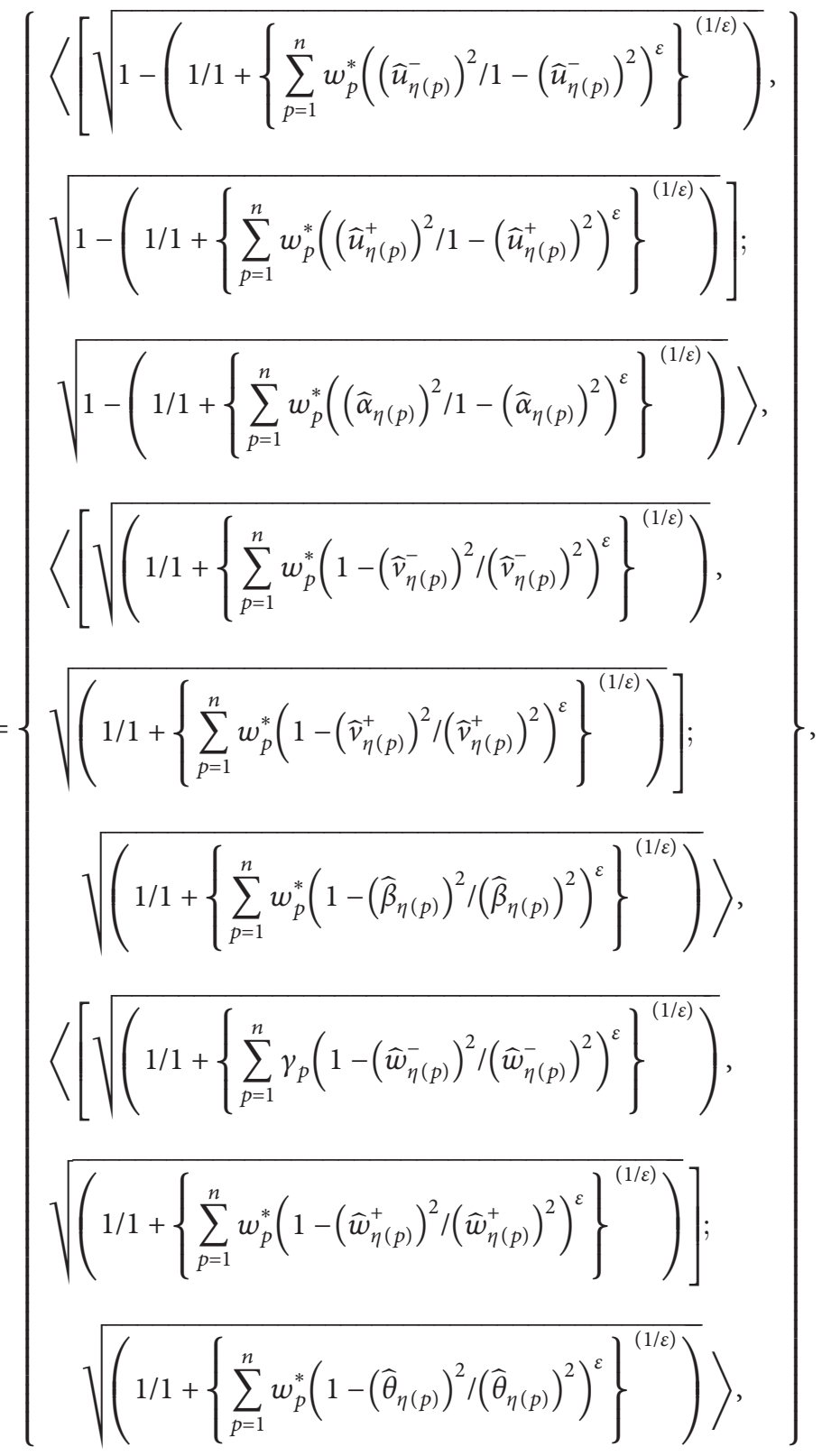


where $\gamma_{p}$ represent the weight vector where $(p=1,2,3, \ldots, n)$ and $\sum_{p=1}^{n} \gamma_{p}=1, \gamma_{p} \geq 0$, and $(p=1,2 \ldots . . n)$, and $p^{\text {th }}$ largest value is $\breve{S}_{\widetilde{x}_{\eta(p)}^{*}}\left(\breve{S}_{\widetilde{x}_{\eta(p)}^{*}}^{*}=n \gamma_{p} \breve{S}_{\tilde{x}_{\eta(p)}^{*}}, p \in N\right)$, and the total order is $\breve{S}_{\widetilde{x}_{\eta(1)}}^{*} \geq \breve{S}_{\widetilde{x}_{\eta(2)}}^{*} \geq \ldots \geq \breve{S}_{\widetilde{x}_{\eta(n)}^{*}}$ where $w_{p}^{*}=\left(w_{1}^{*}, w_{2}^{*}, \ldots, w_{n}^{*}\right)$ and $w_{p}^{*} \geq 0$ and $\sum_{p=1}^{n} w_{p}^{*}=1$.

Proof. The proof is similar to Theorem 1.

The properties of SCFDHWA are as follows:
(1) Idempotency. We have the collection of SCFNs $\widetilde{S}_{\widetilde{x}_{p}}=$ $\left\{x,\left\langle\left[\widehat{u}_{p}^{-}, \widehat{u}_{p}^{+}\right], \widehat{\alpha}_{p}\right\rangle, \quad\left\langle\left[\widehat{v}_{p}^{-}, \widehat{v}_{p}^{+}\right], \widehat{\beta}_{p}\right\rangle,\left\langle\left[\widehat{w}_{p}^{-}, \widehat{w}_{p}^{+}\right], \widehat{\theta}_{p}^{p}\right\rangle\right\}$ $(p=1,2, \ldots, n)$ in $X$. Then, we say collection of SCFNs $\breve{S}_{\widetilde{x}_{p}}(p=1,2, \ldots, n)$ is equal, i.e.,

$$
\operatorname{SCFDHWA}\left(\breve{S}_{\widetilde{x}_{1}}, \breve{S}_{\widetilde{x}_{2}}, \ldots . ., \breve{S}_{\widetilde{x}_{n}}\right)=\breve{S}_{\widetilde{x}} .
$$

(2) Boundary. We have the collection of SCFNs $\breve{S}_{\widetilde{x}_{p}}=$ $\left\{x,\left\langle\left[\widehat{u}_{p}^{-}, \widehat{u}_{p}^{+}\right], \widehat{\alpha}_{p}\right\rangle, \quad\left\langle\left[\widehat{v}_{p}^{-}, \widehat{v}_{p}^{+}\right], \widehat{\beta}_{p}\right\rangle,\left\langle\left[\widehat{w}_{p}^{-}, \widehat{w}_{p}^{+}\right], \widehat{\theta}_{p}^{p}\right\rangle\right\}$ $(p=1,2, \ldots, n)$ in $X$.

$$
\begin{aligned}
\breve{S}_{\tilde{x}_{p}}{ }^{-} & =\left\langle\left(\left[\min _{p} \widehat{u}_{p}^{-}, \min _{p} \widehat{u}_{p}^{+}\right], \min _{p} \widehat{\alpha}_{p}\right),\left(\left[\max _{p} \widehat{v}_{p}^{-}, \max _{p} \widehat{v}_{p}^{+}\right], \max _{p} \widehat{\beta}_{p}\right),\left(\left[\max _{p} \widehat{w}_{p}^{-}, \max _{p} \widehat{w}_{p}^{+}\right], \max _{p} \widehat{\theta}_{p}\right)\right\rangle, \breve{S}_{\widetilde{x}_{p}}^{+} \\
& =\left\langle\left(\left[\max _{p} \widehat{u}_{p}^{-}, \max _{p} \widehat{u}_{p}^{+}\right], \max _{p} \widehat{\alpha}_{p}\right),\left(\left[\min _{p} \widehat{v}_{p}^{-}, \min _{p} \widehat{v}_{p}^{+}\right], \min _{p} \widehat{\beta}_{p}\right),\left(\left[\min _{p} \widehat{w}_{p}^{-}, \min _{p} \widehat{w}_{p}^{+}\right], \min _{p} \widehat{\theta}_{p}\right)\right\rangle .
\end{aligned}
$$

Thus,

$$
\breve{S}_{\widetilde{x}_{p}} \leq \operatorname{SCFDHWA}\left(\breve{S}_{\widetilde{x}_{1}}, \breve{S}_{\widetilde{x}_{2}}, \ldots ., \breve{S}_{\widetilde{x}_{n}}\right) \leq \breve{S}_{\widetilde{x}_{p}}^{+} .
$$

(3) Monotonicity. We have the collection of SCFNs $\breve{S}_{\widetilde{x}_{p}}=$ $\left\langle p_{\widetilde{x}_{p}}(f), \breve{I}_{\widetilde{x}_{p}}(f), \breve{N}_{\widetilde{x}_{p}}(f)\right\rangle(p=1,2 \ldots, n)$ in $X$. $\breve{S}_{\widetilde{x}_{p}} \subseteq \widetilde{S}_{\widetilde{x}_{p}}^{k}$ for $(p=1,2, \ldots, n)$, then $\operatorname{SCFDHWA}\left(\breve{S}_{\widetilde{x}_{1}}, \breve{S}_{\widetilde{x}_{2}}, \ldots, \breve{S}_{\widetilde{x}_{n}}\right) \subseteq \operatorname{SCFDHWA}\left(\breve{S}_{\widetilde{x}_{1}}^{k}, \breve{S}_{\widetilde{x}_{2}}^{k}, \ldots, \breve{S}_{\widetilde{x}_{n}}^{k}\right)$.

4.2. Spherical Cubic Dombi Weighted Geometric Operators. On the basis of Dombi operator of SCFNs, we present the weighted geometric aggregated operations as follows.
Definition 22. We have the collection of SCFNs $\breve{S}_{\widehat{x}_{p}}=\left\{x,\left\langle\left[\widehat{u}_{p}^{-}, \widehat{u}_{p}^{+}\right], \widehat{\alpha}_{p}\right\rangle,\left\langle\left[\widehat{v}_{p}^{-}, \widehat{v}_{p}^{+}\right], \widehat{\beta}_{p}\right\rangle, \quad\left\langle\left[\widehat{w}_{p}^{-}, \widehat{w}_{p}^{+}\right], \widehat{\theta}_{p}\right\rangle\right\}$ where $(p=1,2, \ldots, n)$ in $X$. The spherical cubic fuzzy Dombi weighted geometric (SCFDWG) operator is defined as

$$
\left(\breve{S}_{\widetilde{x}_{1}}, \breve{S}_{\widetilde{x}_{2}}, \breve{S}_{\widetilde{x}_{3}}, \ldots, \breve{S}_{\widetilde{x}_{n}}\right)=\prod_{p=1}^{n}\left(\breve{S}_{\widetilde{x}_{p}}\right)^{\gamma_{p}}
$$

where $\gamma_{p}$ represent the weight vector with $\gamma_{p} \geq 0$ and $\sum_{p=1}^{n} \gamma_{p}=1$.

Theorem 4. We have the collection of SCFNs $\widehat{S}_{\widetilde{x}_{p}}=\left\{x,\left\langle\left[\widehat{u}_{p}^{-}, \widehat{u}_{p}^{+}\right], \widehat{\alpha}_{p}\right\rangle, \quad\left\langle\left[\widehat{v}_{p}^{-}, \widehat{v}_{p}^{+}\right], \widehat{\beta}_{p}\right\rangle,\left\langle\left[\widehat{w}_{p}^{-}, \widehat{w}_{p}^{+}\right], \widehat{\theta}_{p}\right\rangle\right\}$ $(p=1,2, \ldots, n)$ in $X$. Then, the spherical cubic fuzzy Dombi weighted geometric (SCFDWG) operator with $\varepsilon>0$ is defined as

$$
\begin{aligned}
& \operatorname{SCFDWG}\left(\breve{S}_{\widetilde{x}_{1}}, \breve{S}_{\widetilde{x}_{2}}, \ldots, \breve{S}_{\widetilde{x}_{n}}\right)
\end{aligned}
$$

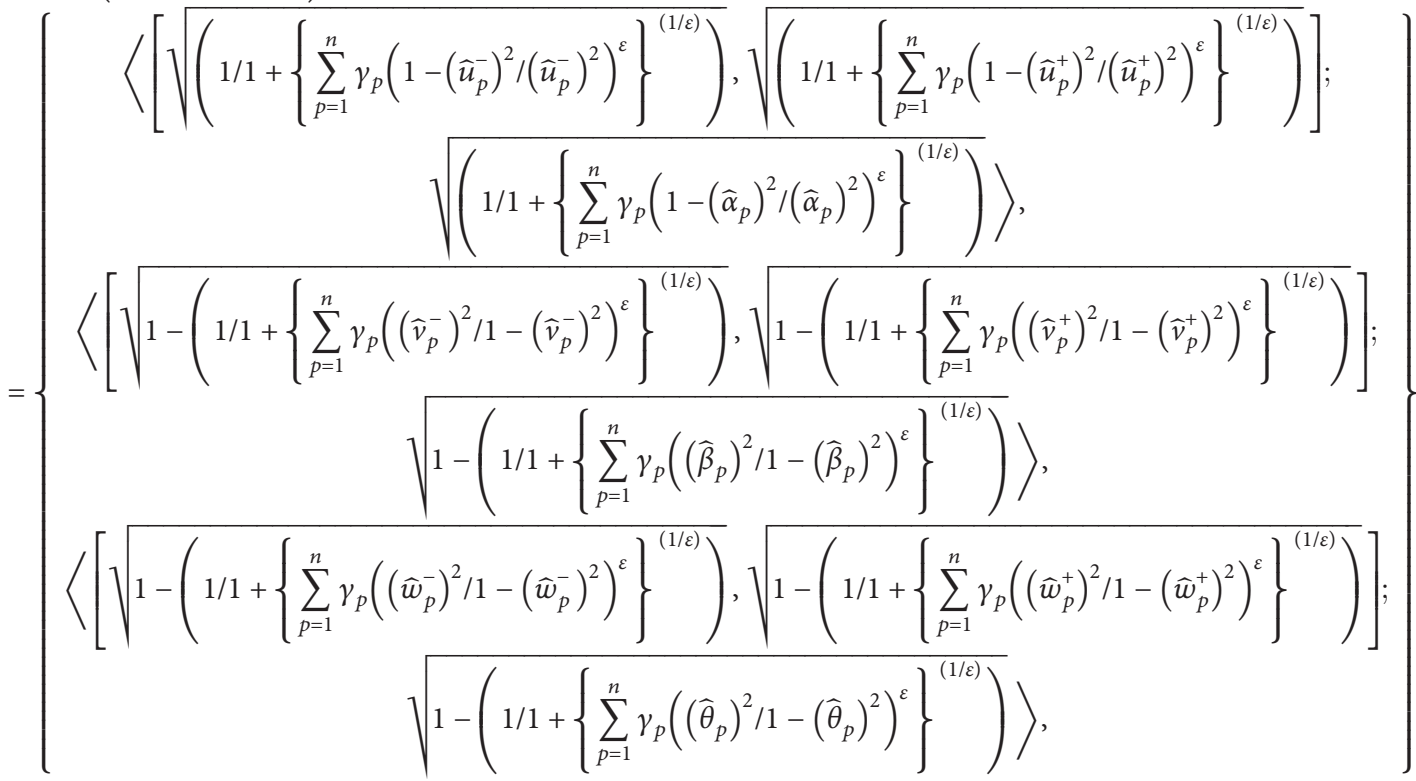


where $\gamma_{p}(p=1,2,3, \ldots, n)$ represent the weight vector so that $\sum_{p=1}^{n} \gamma_{p}=1$ and $\gamma_{p} \geq 0$.

Proof. The proof is similar to Theorem 1.

The properties of SCFDWG are as follows:

(1) Idempotency. We have the collection of SCFNs $\breve{S}_{\widetilde{x}_{p}}=$ $\left\{x,\left\langle\left[\widehat{u}_{p}^{-}, \widehat{u}_{p}^{+}\right], \widehat{\alpha}_{p}\right\rangle,\left\langle\left[\widehat{v}_{p}^{-}, \widehat{v}_{p}^{+}\right], \quad \widehat{\beta}_{p}\right\rangle,\left\langle\left[\widehat{w}_{p}^{-}, \widehat{w}_{p}^{+}\right], \widehat{\theta}_{p}^{p}\right\rangle\right\}$ $(p=1,2, \ldots, n)$ in $X$. Then, we say collection of SCFNs $S_{\tilde{x}_{p}}(p=1,2, \ldots, n)$ is equal, i.e.,

$$
\operatorname{SCFDWG}\left(\breve{S}_{\widetilde{x}_{1}}, \breve{S}_{\widetilde{x}_{2}}, \ldots, \breve{S}_{\widetilde{x}_{n}}\right)=\breve{S}_{\widetilde{x}} \text {. }
$$

(2) Boundary. We have the collection of SCFNs $\breve{S}_{\widetilde{x}_{p}}=$ $\left\{x,\left\langle\left[\widehat{u}_{p}^{-}, \widehat{u}_{p}^{+}\right], \widehat{\alpha}_{p}\right\rangle, \quad\left\langle\left[\widehat{v}_{p}^{-}, \widehat{v}_{p}^{+}\right], \widehat{\beta}_{p}\right\rangle,\left\langle\left[\widehat{w}_{p}^{-}, \widehat{w}_{p}^{+}\right], \widehat{\theta}_{p}^{p}\right\rangle\right\}$ $(p=1,2, \ldots, n)$ in $X$.

$$
\begin{aligned}
& \breve{S}_{\widetilde{x}_{p}}^{-}=\left\langle\left(\left[\min _{p} \widehat{u}_{p}^{-}, \min _{p} \widehat{u}_{p}^{+}\right], \min _{p} \widehat{\alpha}_{p}\right),\left(\left[\max _{p} \widehat{v}_{p}^{-}, \max _{p} \widehat{v}_{p}^{+}\right], \max _{p} \widehat{\beta}_{p}\right),\left(\left[\max _{p} \widehat{w}_{p}^{-}, \max _{p} \widehat{w}_{p}^{+}\right], \max _{p} \widehat{\theta}_{p}\right)\right\rangle, \\
& \stackrel{S}{S}_{\widetilde{x}_{p}}^{+}=\left\langle\left(\left[\max _{p} \widehat{u}_{p}^{-}, \max _{p} \widehat{u}_{p}^{+}\right], \max _{p} \widehat{\alpha}_{p}\right),\left(\left[\min _{p} \widehat{v}_{p}^{-}, \min _{p} \widehat{v}_{p}^{+}\right], \min _{p} \widehat{\beta}_{p}\right),\left(\left[\min _{p} \widehat{w}_{p}^{-}, \min _{p} \widehat{w}_{p}^{+}\right], \min _{p} \widehat{\theta}_{p}\right)\right\rangle .
\end{aligned}
$$

Thus, $\breve{S}_{\widetilde{x}_{p}}^{-} \leq \operatorname{SCFDWG}\left(\breve{S}_{\widetilde{x}_{1}}, \breve{S}_{\widetilde{x}_{2}}, \ldots, \breve{S}_{\widetilde{x}_{n}}\right) \leq \breve{S}_{\widetilde{x}_{p}}^{+}$.

(3) Monotonicity. We have the collection of SCFNs $\widetilde{S}_{\widetilde{x}_{p}}=$ $\left\{x,\left\langle\left[\widehat{u}_{p}^{-}, \widehat{u}_{p}^{+}\right], \widehat{\alpha}_{p}\right\rangle, \quad\left\langle\left[\widehat{v}_{p}^{-}, \widehat{v}_{p}^{+}\right], \widehat{\beta}_{p}\right\rangle,\left\langle\left[\widehat{w}_{p}^{-}, \widehat{w}_{p}^{+}\right], \widehat{\theta}_{p}\right\rangle\right\}$ $(p=1,2, \ldots, n)$ in $X . \breve{S}_{\widetilde{x}_{p}} \subseteq \breve{S}_{\widetilde{x}_{p}}^{k}$ for $(p=1,2, \ldots, n)$, then

$$
\operatorname{SCFDWG}\left(\breve{S}_{\widetilde{x}_{1}}, \breve{S}_{\widetilde{x}_{2}}, \ldots, \breve{S}_{\widetilde{x}_{n}}\right) \subseteq \operatorname{SCFDWG}\left(\breve{S}_{\widetilde{x}_{1}}^{k}, \breve{S}_{\widetilde{x}_{2}}^{k}, \ldots, \breve{S}_{\widetilde{x}_{n}}^{k}\right)
$$

Definition 23. We have the collection of SCFNs $\widehat{S}_{\widetilde{x}_{p}}=\left\{x,\left\langle\left[\widehat{u}_{p}^{-}, \widehat{u}_{p}^{+}\right], \widehat{\alpha}_{p}\right\rangle,\left\langle\left[\widehat{v}_{p}^{-}, \widehat{v}_{p}^{+}\right], \widehat{\beta}_{p}\right\rangle,\left\langle\left[\widehat{w}_{p}^{-}, \widehat{w}_{p}^{+}\right], \widehat{\theta}_{p}\right\rangle\right\}$ where $(p=1,2, \ldots, n)$ in $X$. The spherical cubic fuzzy Dombi ordered weighted geometric (SCFDOWG) operator is

$$
\operatorname{SCFDOWG}\left(\breve{S}_{\widetilde{x}_{1}}, \breve{S}_{\widetilde{x}_{2}}, \breve{S}_{\widetilde{x}_{3}}, \ldots, \breve{S}_{\widetilde{x}_{n}}\right)=\prod_{p=1}^{n}\left(\breve{S}_{\widetilde{x}_{\eta_{(p)}}}\right)^{\gamma_{p}},
$$

where $\gamma_{p}$ represents the weight vector with $\gamma_{p} \geq 0$, and $\sum_{p=1}^{n} \gamma_{p}=1$, and the largest weighted value is $S_{\widetilde{x}_{\eta_{(p)}}}$, and the total order is $S_{\widetilde{x}_{\eta_{(1)}}} \geq S_{\widetilde{x}_{\eta_{(2)}}} \geq \ldots \geq S_{\widetilde{x}_{\eta_{(n)}}}$.

Theorem 5. We have the collection of SCFNs $\widehat{S}_{\widetilde{x}_{p}}=\left\{x,\left\langle\left[\widehat{u}_{p}^{-}, \widehat{u}_{p}^{+}\right], \widehat{\alpha}_{p}\right\rangle,\left\langle\left[\widehat{v}_{p}^{-}, \widehat{v}_{p}^{+}\right], \widehat{\beta}_{p}\right\rangle,\left\langle\left[\widehat{w}_{p}^{-}, \widehat{w}_{p}^{+}\right], \widehat{\theta}_{p}\right\rangle\right\}$ where $(p=1,2, \ldots, n)$ in $X$. The spherical cubic fuzzy Dombi ordered weighted geometric (SCFDOWG) operator is defined as 


$$
\begin{aligned}
& \operatorname{SCFDOWG}\left(\breve{S}_{\widetilde{x}_{1}}, \breve{S}_{\widetilde{x}_{2}}, \breve{S}_{\widetilde{x}_{3}}, \ldots, \breve{S}_{\widetilde{x}_{n}}\right)
\end{aligned}
$$

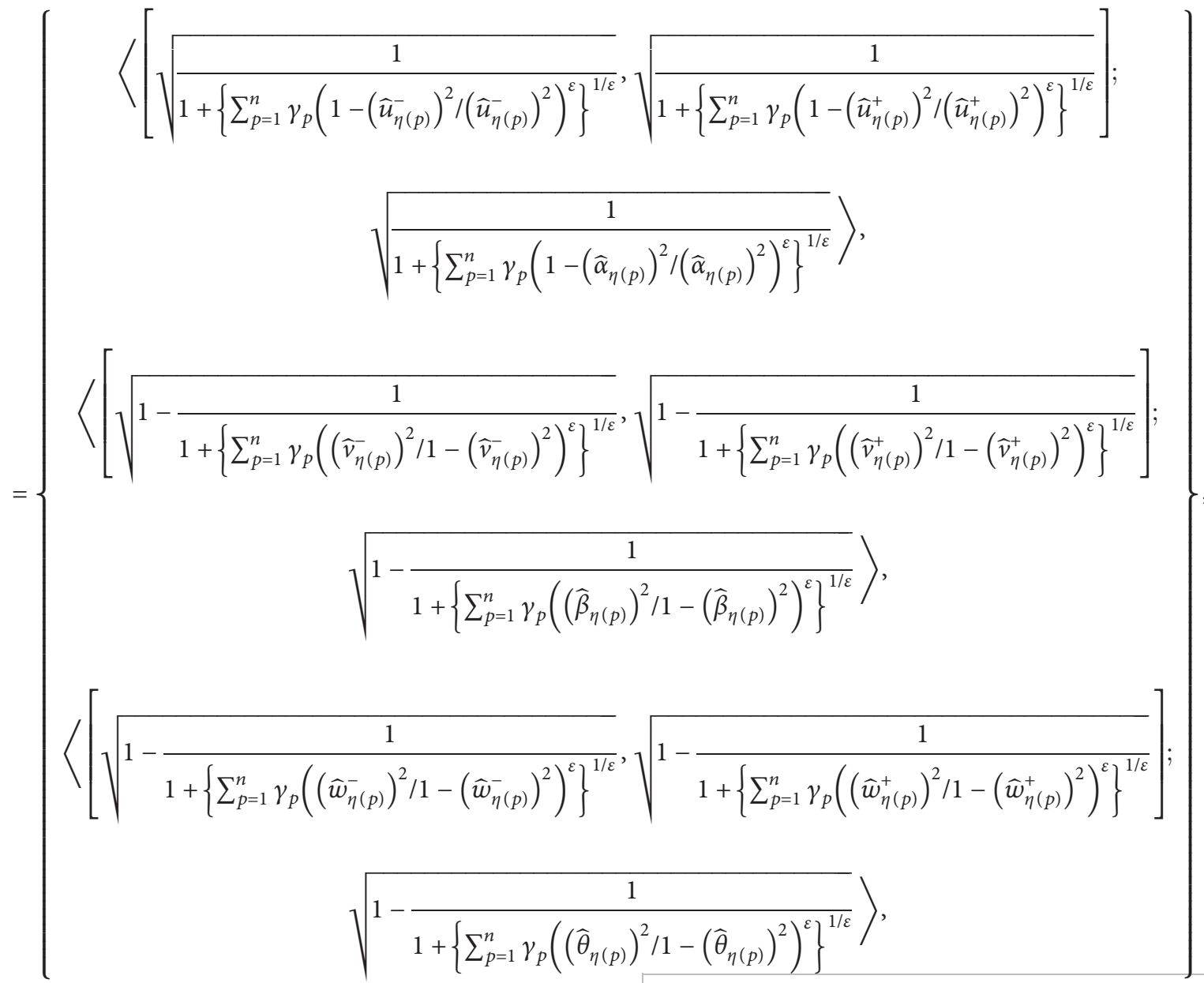

where the weight vector is represented as $\gamma_{p}$ with $\sum_{p=1}^{n} \gamma_{p}=1$ and $\gamma_{p} \geq 0$ where $(p=1,2, \ldots, n)$, and the largest weighted value is $S_{\widetilde{x}_{\eta}}$, and the total order is $\widetilde{S}_{\widetilde{x}_{\eta_{(1)}}} \geq \breve{S}_{\widetilde{x}_{\eta_{(2)}}} \geq, \ldots, \geq \widetilde{S}_{\widetilde{x}_{\eta_{(n)}}}$.

Proof. The proof is similar to Theorem 4.

The properties of SCFDOWG are as follows:

(1) Idempotency. We have the collection of SCFNs $\breve{S}_{\widetilde{x}_{p}}=$ $\left\{x,\left\langle\left[\widehat{u}_{p}^{-}, \widehat{u}_{p}^{+}\right], \widehat{\alpha}_{p}\right\rangle,\left\langle\left[\widehat{v}_{p}^{-}, \widehat{v}_{p}^{+}\right], \widehat{\beta}_{p}\right\rangle,\left\langle\left[\widehat{w}_{p}^{-}, \widehat{w}_{p}^{+}\right], \widehat{\theta}_{p}^{p}\right\rangle\right\}$ $(p=1,2, \ldots, n)$ in $X$. Then, we say collection of SCFNs $S_{\widetilde{x}_{p}}(p=1,2, \ldots, n)$ is equal, i.e.,

$$
\operatorname{SCFDOWG}\left(\breve{S}_{\widetilde{x}_{1}}, \breve{S}_{\widetilde{x}_{2}}, \ldots, \breve{S}_{\widetilde{x}_{n}}\right)=\breve{S}_{\widetilde{x}}
$$

(2) Boundary. We have the collection of SCFNs $\breve{S}_{\widetilde{x}}=$ $\left\{x,\left\langle\left[\widehat{u}_{p}^{-}, \widehat{u}_{p}^{+}\right], \widehat{\alpha}_{p}\right\rangle, \quad\left\langle\left[\widehat{v}_{p}^{-}, \widehat{v}_{p}^{+}\right], \widehat{\beta}_{p}\right\rangle,\left\langle\left[\widehat{w}_{p}^{-}, \widehat{w}_{p}^{+}\right], \widehat{\theta}_{p}^{p}\right\rangle\right\}$ $(p=1,2, \ldots, n)$ in $X$.

$$
\begin{aligned}
& \breve{S}_{\widetilde{x}_{p}^{-}}=\left\langle\left(\left[\min _{p} \widehat{u}_{p}^{-}, \min _{p} \widehat{u}_{p}^{+}\right], \min _{p} \widehat{\alpha}_{p}\right),\left(\left[\max _{p} \widehat{v}_{p}^{-}, \max _{p} \widehat{v}_{p}^{+}\right], \max _{p} \widehat{\beta}_{p}\right),\left(\left[\max _{p} \widehat{w}_{p}^{-}, \max _{p} \widehat{w}_{p}^{+}\right], \max _{p} \widehat{\theta}_{p}\right)\right\rangle, \\
& \breve{S}_{\widetilde{x}_{p}{ }^{+}=}\left\langle\left(\left[\max _{p} \widehat{u}_{p}^{-}, \max _{p} \widehat{u}_{p}^{+}\right], \max _{p} \widehat{\alpha}_{p}\right),\left(\left[\min _{p} \widehat{v}_{p}^{-}, \min _{p} \widehat{v}_{p}^{+}\right], \min _{p} \widehat{\beta}_{p}\right),\left(\left[\min _{p} \widehat{w}_{p}^{-}, \min _{p} \widehat{w}_{p}^{+}\right], \min _{p} \widehat{\theta}_{p}\right)\right\rangle .
\end{aligned}
$$

Thus,

$$
\breve{S}_{\widetilde{x}_{p}}^{-} \leq \operatorname{SCFDOWG}\left(\breve{S}_{\widetilde{x}_{1}}, \breve{S}_{\widetilde{x}_{2}}, \ldots, \breve{S}_{\widetilde{x}_{n}}\right) \leq \breve{S}_{\widetilde{x}_{p}}^{+}
$$

(3) Monotonicity. We have the collection of SCFNs $\breve{S}_{\widetilde{x}_{p}}=$ $\left\{x,\left\langle\left[\widehat{u}_{p}^{-}, \widehat{u}_{p}^{+}\right], \widehat{\alpha}_{p}\right\rangle,\left\langle\left[\widehat{v}_{p}^{-}, \widehat{v}_{p}^{+}\right], \widehat{\beta}_{p}\right\rangle,\left\langle\left[\widehat{w}_{p}^{-}, \widehat{w}_{p}^{+}\right], \widehat{\theta}_{p}\right\rangle\right\} \quad(p=$ $1,2, \ldots, n)$ in $X . \breve{S}_{\widetilde{x}_{p}} \subseteq S_{\widetilde{x}_{p}}^{k}$ for $(p=1,2, \ldots, n)$, then 
$\operatorname{SCFDOWG}\left(\breve{S}_{\widetilde{x}_{1}}, \breve{S}_{\widetilde{x}_{2}}, \ldots, \breve{S}_{\widetilde{x}_{n}}\right) \subseteq \operatorname{SCFDOWG}\left(\breve{S}_{\widetilde{x}_{1}}^{k}, \breve{S}_{\widetilde{x}_{2}}^{k}, \ldots, \breve{S}_{\widetilde{x}_{n}}^{k}\right)$.

Definition 24. We have the collection of SCFNs $S_{\widetilde{x}_{p}}=\left\{x,\left\langle\left[\widehat{u}_{p}^{-}, \widehat{u}_{p}^{+}\right], \widehat{\alpha}_{p}\right\rangle,\left\langle\left[\widehat{v}_{p}^{-}, \widehat{v}_{p}^{+}\right], \widehat{\beta}_{p}\right\rangle,\left\langle\left[\widehat{w}_{p}^{-}, \widehat{w}_{p}^{+}\right], \widehat{\theta}_{p}\right\rangle\right\}$

where $(p=1,2, \ldots, n)$ in $X$. The spherical cubic fuzzy Dombi hybrid weighted geometric (SCFDHWG) operator is defined as

$$
\left(\breve{S}_{\widetilde{x}_{1}}, \breve{S}_{\widetilde{x}_{2}}, \breve{S}_{\widetilde{x}_{3}}, \ldots, \breve{S}_{\widetilde{x}_{n}}\right)=\prod_{p=1}^{n}\left(\breve{S}_{\widetilde{x}_{p}}^{*}\right)^{\gamma_{p}},
$$

where $\gamma_{p}$ represents the weight vector with $\gamma_{p} \geq 0$, and

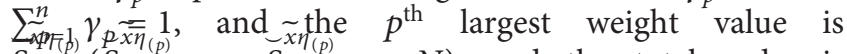
$S_{*}\left(S_{*}^{(p)}=n \gamma_{p} S_{*}, p \in N\right)$, and the total order is $S_{\widetilde{x}_{n}} \geq S_{\widetilde{x}_{n}} \geq, \ldots, \geq \widetilde{S}_{\widetilde{x}_{n}}$, and $w=\left(w_{1}, w_{2}, \ldots, w_{n}\right)$ represent the weights with $\sum_{p=1}^{n} w_{p}^{*}=1, w_{p}^{*} \geq 0$.

Theorem 6. We have the collection of SCFNs $\widetilde{S}_{\widehat{x}_{p}}=\left\{x,\left\langle\left[\widehat{u}_{p}^{-}, \widehat{u}_{p}^{+}\right], \widehat{\alpha}_{p}\right\rangle,\left\langle\left[\widehat{v}_{p}^{-}, \widehat{v}_{p}^{+}\right], \widehat{\beta}_{p}\right\rangle,\left\langle\left[\widehat{w}_{p}^{-}, \widehat{w}_{p}^{+}\right], \widehat{\theta}_{p}\right\rangle\right\}$ where $(p=1,2, \ldots, n)$ in $X$. The spherical cubic fuzzy Dombi hybrid weighted geometric (SCFDHWG) operator is defined as

$$
\begin{aligned}
& \operatorname{SCFDHWG}\left(\breve{S}_{\widetilde{x}_{1}}, \breve{S}_{\widetilde{x}_{2}}, \breve{S}_{\widetilde{x}_{3}}, \ldots, \breve{S}_{\widetilde{x_{n}}}\right)
\end{aligned}
$$

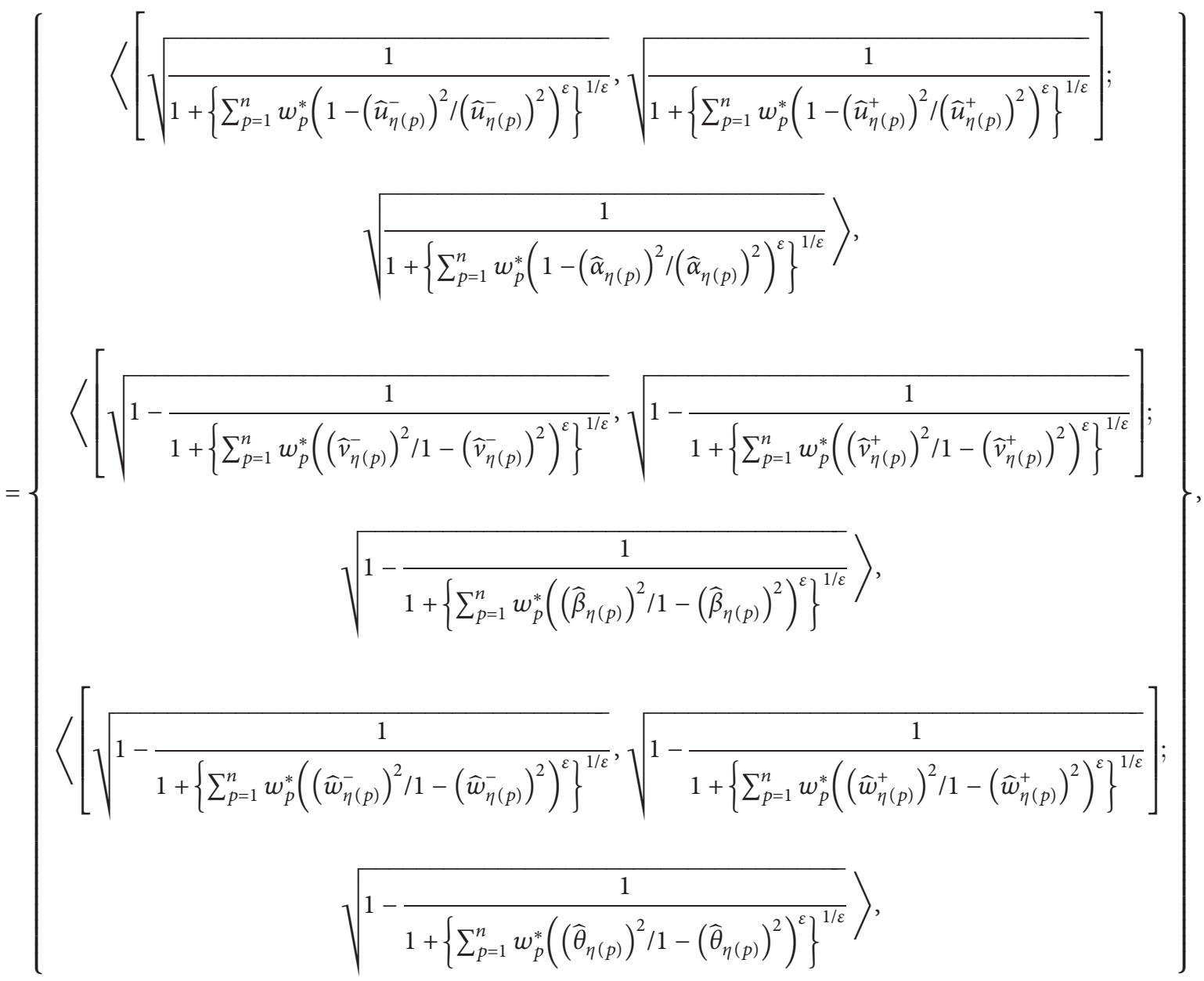

where $\gamma_{p}$ represent the weight vector with $\gamma_{p} \geq 0$, and $\sum_{x p=1}^{n} \gamma_{p}=1$, and $\tilde{-}_{x \eta}$ the $p^{\text {th }}$ largest weight value is $\underline{S}_{*}\left(S_{*^{*}}=n \gamma_{p} S_{*}, p \in N\right)$, and the total order is $S_{\widetilde{x}_{\eta^{n}(1)}} \geq S_{\widetilde{x}_{\eta^{\prime}(2)}} \geq \ldots \geq S_{\widetilde{x}_{\eta(n)}}$, and $w_{p}^{*} \geq 0, \sum_{p=1}^{n} w_{p}^{*}=1$, $w_{p}^{* *(1)}=\left(w_{1}^{*}, w_{2}^{*}, \ldots, w_{n}^{*}\right)^{*}$
Proof. The proof is similar to Theorem 4 .

The properties of SCFDWG are as follows:

(1) Idempotency. We have the collection of SCFNs $\widetilde{S}_{\widetilde{x}}=$ $\left\{x,\left\langle\left[\widehat{u}_{p}^{-}, \widehat{u}_{p}^{+}\right], \quad \widehat{\alpha}_{p}\right\rangle,\left\langle\left[\widehat{v}_{p}^{-}, \widehat{v}_{p}^{+}\right], \widehat{\beta}_{p}\right\rangle,\left\langle\left[\widehat{w}_{p}^{-}, \widehat{w}_{p}^{+}\right], \widehat{\theta}_{p}^{p}\right\rangle\right\}$ 
$(p=1,2, \ldots, n)$ in $X$. Then, we say collection of SCFNs $S_{\tilde{x}_{p}}(p=1,2, \ldots, n)$ is equal, i.e.,

$$
\operatorname{SCFDHWG}\left(\breve{S}_{\widetilde{x}_{1}}, \breve{S}_{\widetilde{x}_{2}}, \ldots, \breve{S}_{\widetilde{x}_{n}}\right)=\breve{S}_{\widetilde{x}} .
$$

(2) Boundary. We have the collection of SCFNs $\breve{S}_{\widetilde{x}}=$ $\left\{x,\left\langle\left[\widehat{u}_{p}^{-}, \widehat{u}_{p}^{+}\right], \quad \widehat{\alpha}_{p}\right\rangle,\left\langle\left[\widehat{v}_{p}^{-}, \widehat{v}_{p}^{+}\right], \widehat{\beta}_{p}\right\rangle,\left\langle\left[\widehat{w}_{p}^{-}, \widehat{w}_{p}^{+}\right], \widehat{\theta}_{p}^{p}\right\rangle\right\}$ $(p=1,2, \ldots, n)$ in $X$.

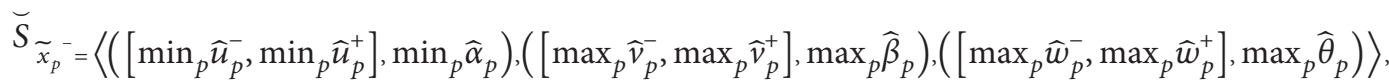

$$
\begin{aligned}
& \breve{S}_{\widetilde{x}_{p}^{+}}=\left\langle\left(\left[\max _{p} \widehat{u}_{p}^{-}, \max _{p} \widehat{u}_{p}^{+}\right], \max _{p} \widehat{\alpha}_{p}\right),\left(\left[\min _{p} \widehat{v}_{p}^{-}, \min _{p} \widehat{v}_{p}^{+}\right], \min _{p} \widehat{\beta}_{p}\right),\left(\left[\min _{p} \widehat{w}_{p}^{-}, \min _{p} \widehat{w}_{p}^{+}\right], \min _{p} \widehat{\theta}_{p}\right)\right\rangle .
\end{aligned}
$$

Thus,

$$
\breve{S}_{\widetilde{x}_{p}}^{-} \leq \operatorname{SCFDHWG}\left(\breve{S}_{\widetilde{x}_{1}}, \breve{S}_{\widetilde{x}_{2}}, \ldots, \breve{S}_{\widetilde{x}_{n}}\right) \leq \breve{S}_{\widetilde{x}_{p}}^{+}
$$

(3) Monotonicity. We have the collection of SCFNs $\breve{S}_{\widetilde{x}_{p}}=$ $\left\langle\breve{P}_{\widetilde{x}_{p}}(f), \breve{I}_{\widetilde{x}_{p}}(f), \widetilde{N}_{\widetilde{x}_{p}}(f)\right\rangle(p=1,2, \ldots, n)$ in $X$. $\breve{S}_{\widetilde{x}_{p}} \subseteq \breve{S}_{\widetilde{x}_{p}}^{k}$ for $(p=1,2, \ldots, n)$, then $\operatorname{SCFDHWG}\left(\breve{S}_{\widetilde{x}_{1}}, \breve{S}_{\widetilde{x}_{2}}, \ldots, \breve{S}_{\widetilde{x}_{n}}\right) \subseteq \operatorname{SCFDHWG}\left(\breve{S}_{\widetilde{x}_{1}}^{k}, \breve{S}_{\widetilde{x}_{x}}^{k}, \ldots, \breve{S}_{\widetilde{x}_{n}}^{k}\right)$.

\section{Computation for MAGDM Using Spherical Cubic Fuzzy Sets}

Now, we present a new method to decision-making through the idea of spherical cubic fuzzy set. This methodology will utilize the data characterized by the given problem and only need not to bother with any extra data given by the decision makers, so as to avoid the impact of information impacting on the decision results. In the following, we will introduce a spherical cubic fuzzy set decision-making matrix as indicated below.

Let $A=\left(a_{1}, a_{2}, \ldots, a_{m}\right)$ be a collection of $m$ appropriate alternatives and $B=\left(b_{1}, b_{2}, \ldots, b_{n}\right)$ be a definite collection of $n$ criterion, where $a_{i}$ specify the $i^{\text {th }}$ criteria. Let $C=\left(c_{1}, c_{2}, \ldots, c_{s}\right)$ be a finite set of $s$ criteria, where $c_{s}$ indicate the $s^{\text {th }}$ expert. The analyst $c_{k}$ supplies here evaluation of an alternative $a_{i}$ on an attribute $b_{j}$ as SCFNs $(i=1,2, \ldots, m, j=1,2 \ldots, n)$. The analyst data are depicted by spherical cubic fuzzy set decision matrix $D^{s}=\left[\left(E_{i p}^{-(s)}, E_{i p}^{+(s)}\right), N_{i p}^{-(s)}\right]_{m \times n}$. Suppose that $\gamma_{p}$ represents the weight vector of the attribute $b_{j}$ that $\sum_{p=1}^{n} \gamma_{p}=1$ where $0 \leq \gamma_{p} \leq 1$, and $\phi=\left(\phi_{1}, \phi_{2}, \ldots, \phi_{m}\right)$ is the weight vector of the $c_{k}$ decision makers so that $\sum_{k=1}^{n} \phi_{k}=1, \phi_{k} \leq 1$ as we make the spherical cubic fuzzy decision maker matrices $D^{s}=\left[\left(E_{i p}^{-(s)}, E_{i p}^{+(s)}\right), N_{i p}^{-(s)}\right]_{m \times n}$ for decision. Generally, criteria have two sorts. One is a profit criteria, and other is cost criteria. Thus, the spherical cubic fuzzy decision matrices having cost criteria matrices $D^{s}=\left[\left(E_{i p}^{-(s)}, E_{i p}^{+(s)}\right), N_{i p}^{-(s)}\right]_{m \times n}$ might be transformed into normalized spherical cubic fuzzy decision matrices $\gamma^{s}=\left[\left(\gamma_{i p}^{-(s)}, \gamma_{i p}^{+(s)}\right), \widehat{w}_{i p}^{-(s)}\right]_{m \times n}$ where $\gamma_{i p}^{-(s)}=$
$\left\{\begin{array}{c}E_{i p}^{-(s)}, \text { for benefit criteria } \\ \widetilde{E}_{i p}^{-(s)} \text {, for cost criteria }\end{array}\right\} \widetilde{E}_{i p}^{-(s)}$ represents the negation of $E_{i p}^{-(s)}$. If all the criteria have same type, then no need to normalization.

Step 1. Make the spherical cubic fuzzy decision matrix $D=\left(E_{i p}\right)_{m \times n}=\left[\left(E_{i p}^{-(s)}, E_{i p}^{+(s)}\right), N_{i p}^{-(s)}\right]_{m \times n}$ and then use the idea of SCFWA/SCFWG operator, and the aggregated spherical cubic fuzzy value $\gamma_{i}^{k}$ of the alternative $a_{i}$ is in the following way.

$\gamma_{i}^{k}=\operatorname{SCFWA}_{\gamma}\left[\left(\gamma_{i p}^{k}, \gamma_{i p}^{\prime k}\right), \gamma_{i p}^{\prime \prime-(s)}\right]_{m \times n}$ where weight vector is represented as $\gamma=\left(\gamma_{1}, \gamma_{2}, \ldots, \gamma_{n}\right)$; we apply the SCFWA, SCFOWA, SCFWG, SCFOWG, SCFHWA, and SCFHWG operations to MAGDM which are describeed in the following steps.

Step 2. In view of this, we take the spherical cubic fuzzy data by utilizing the defined Dombi operators to develope the preferable value of the alternative with the weights $w=\left(w_{1}, w_{2}, \ldots, w_{n}\right)$ with $\sum_{p=1}^{n} \gamma_{p}=1$ and $w_{p} \geq 0$.

Step 3. We will find the score and accuracy function of overall preference value $a_{i}(i=1,2, \ldots, m)$.

Step 4. By definition of score function, rank the alternatives $a_{i}(i=1,2, \ldots, m)$ and select the best alternative which has the maximum value of score function.

\section{Numerical Application}

Suppose that finance administrator Mr. A in a wealth administration is evaluating four feasible investing $A_{1}, A_{2}, A_{3}, A_{4}$ (let $\left.A=\left\{a_{1}, a_{2}, a_{3}, a_{4}\right\}\right)$. The firm state that the finance administrator has to assess the following three criteria $B_{1}, B_{2}, B_{3}$ (let $B=\left\{b_{1}, b_{2}, b_{3}\right\}$ ) where $b_{1}$ represents the "highrisk," $b_{2}$ represents the "progress," and $b_{3}$ represents the "Surrounding affects." For the parameter $b_{i} \in B(i=1,2,3)$, Mr. A calls on three skilled group to appraise if $A_{j}(j=$ $1,2,3,4)$ is the best opportunity of potential investment in view of $b_{i}$ and the decision maker has the weights $(0.2,0.3,0.5)$. We will establish the spherical cubic fuzzy set, and decision matrices are represented in Tables 1-3.

Here, $B_{1}$ and $B_{3}$ are cost-type and $B_{2}$ represents profit criteria. Now, we have to normalize the matrices. Normalized data are shown in Tables 4-6. 
TABLE 1: Investing capacity in a wealth administration firm $\mathrm{D}^{1}$.

\begin{tabular}{|c|c|c|c|}
\hline & $b_{1}$ & $b_{2}$ & $b_{3}$ \\
\hline$A_{1}$ & $\left(\begin{array}{c}([0.3,0.4], 0.5), \\
([0.1,0.3], 0.2), \\
([0.1,0.2], 0.2)\end{array}\right)$ & $\left(\begin{array}{c}([0.3,0.4], 0.3), \\
([0.1,0.2], 0.4), \\
([0.2,0.1], 0.2)\end{array}\right)$ & $\left(\begin{array}{c}([0.4,0.5], 0.4), \\
([0.2,0.1], 0.2), \\
([0.2,0.1], 0.4)\end{array}\right)$ \\
\hline$A_{2}$ & $\left(\begin{array}{c}([0.2,0.6], 0.1), \\
([0.2,0.1], 0.2), \\
([0.1,0.2], 0.4)\end{array}\right)$ & $\left(\begin{array}{c}([0.3,0.1], 0.1), \\
([0.3,0.2], 0.2), \\
([0.2,0.4], 0.3)\end{array}\right)$ & $\left(\begin{array}{c}([0.2,0.3], 0.4), \\
([0.3,0.4], 0.2), \\
([0.1,0.3], 0.2)\end{array}\right)$ \\
\hline$A_{3}$ & $\begin{array}{c}\left(\begin{array}{c}([0.4,0.4], 0.2), \\
([0.2,0.1], 0.5), \\
([0.1,0.4], 0.1)\end{array}\right) \\
([0.3,0.3], 0.4),\end{array}$ & $\begin{array}{c}\left(\begin{array}{c}([0.5,0.1], 0.2), \\
([0.1,0.2], 0.3), \\
([0.1,0.2], 0.3)\end{array}\right) \\
([0.5,0.4], 0.2),\end{array}$ & $\left.\begin{array}{c}([0.2,0.3], 0.4), \\
([0.1,0.2], 0.3), \\
([0.1,0.4], 0.2)\end{array}\right)$ \\
\hline$A_{4}$ & $\left(\begin{array}{c}([0.3,0.4], 0.3), \\
([0.3,0.1], 0.2)\end{array}\right)$ & $\left(\begin{array}{c}([0.1,0.2], 0.4), \\
([0.3,0.1], 0.3)\end{array}\right)$ & $\left(\begin{array}{c}([0.2,0.1], 0.3) \\
([0.3,0.2], 0.1)\end{array}\right)$ \\
\hline
\end{tabular}

TABLE 2: Investing capacity in a wealth administration firm $\mathrm{D}^{2}$.

\begin{tabular}{|c|c|c|c|}
\hline & $b_{1}$ & $b_{2}$ & $b_{3}$ \\
\hline$A_{1}$ & $\left(\begin{array}{c}([0.1,0.2], 0.2), \\
([0.2,0.3], 0.5), \\
([0.4,0.1], 0.2)\end{array}\right)$ & $\left(\begin{array}{c}([0.2,0.3], 0.3), \\
([0.4,0.1], 0.1), \\
([0.2,0.2], 0.2)\end{array}\right)$ & $\left(\begin{array}{c}([0.3,0.4], 0.6), \\
([0.1,0.3], 0.2), \\
([0.2,0.2], 0.1)\end{array}\right)$ \\
\hline$A_{2}$ & $\left(\begin{array}{c}([0.4,0.2], 0.6), \\
([0.3,0.1], 0.1), \\
([0.2,0.1], 0.2)\end{array}\right)$ & $\left(\begin{array}{c}([0.4,0.3], 0.1) \\
([0.2,0.1], 0.2) \\
([0.4,0.1], 0.2)\end{array}\right)$ & $\left(\begin{array}{c}([0.2,0.5], 0.8), \\
([0.1,0.3], 0.1), \\
([0.1,0.2], 0.1)\end{array}\right)$ \\
\hline$A_{3}$ & $\left(\begin{array}{c}([0.2,0.4], 0.3), \\
([0.3,0.1], 0.2), \\
([0.3,0.2], 0.1)\end{array}\right)$ & $\left(\begin{array}{c}([0.2,0.1], 0.4), \\
([0.3,0.1], 0.3), \\
([0.1,0.2], 0.2)\end{array}\right)$ & $\left(\begin{array}{c}([0.3,0.5], 0.4), \\
([0.2,0.1], 0.1), \\
([0.3,0.2], 0.3)\end{array}\right)$ \\
\hline$A_{4}$ & $\left(\begin{array}{c}([0.3,0.4], 0.3), \\
([0.1,0.2], 0.1), \\
([0.3,0.1], 0.4)\end{array}\right)$ & $\left(\begin{array}{c}([0.6,0.1], 0.6), \\
([0.1,0.2], 0.1), \\
([0.2,0.1], 0.3)\end{array}\right)$ & $\left(\begin{array}{c}([0.4,0.1], 0.2), \\
([0.1,0.4], 0.3), \\
([0.1,0.2], 0.3)\end{array}\right)$ \\
\hline
\end{tabular}

TABle 3: Investing capacity in a wealth administration firm $\mathrm{D}^{3}$.

\begin{tabular}{|c|c|c|c|}
\hline & $b_{1}$ & $b_{2}$ & $b_{3}$ \\
\hline$A_{1}$ & $\left(\begin{array}{c}([0.4,0.2], 0.3), \\
([0.1,0.2], 0.2), \\
([0.4,0.1], 0.1)\end{array}\right)$ & $\left(\begin{array}{c}([0.4,0.4], 0.3), \\
([0.2,0.4], 0.4), \\
([0.2,0.1], 0.2)\end{array}\right)$ & $\left(\begin{array}{c}([0.1,0.4], 0.6), \\
([0.2,0.3], 0.2), \\
([0.1,0.3], 0.1)\end{array}\right)$ \\
\hline$A_{2}$ & $\left(\begin{array}{c}([0.2,0.5], 0.3), \\
([0.2,0.1], 0.1), \\
([0.1,0.2], 0.4)\end{array}\right)$ & $\left(\begin{array}{c}([0.1,0.5], 0.4), \\
([0.3,0.2], 0.1), \\
([0.2,0.1], 0.3)\end{array}\right)$ & $\left(\begin{array}{c}([0.4,0.2], 0.7), \\
([0.4,0.3], 0.1), \\
([0.2 .0 .1], 0.2)\end{array}\right)$ \\
\hline$A_{3}$ & $\left(\begin{array}{c}([0.5,0.4], 0.6), \\
([0.2,0.3], 0.1) \\
([0.2,0.1], 0.2)\end{array}\right)$ & $\left(\begin{array}{c}([0.2,0.3], 0.1), \\
([0.3,0.4], 0.1), \\
([0.3,0.1], 0.3)\end{array}\right)$ & $\left(\begin{array}{c}([0.3,0.1], 0.2), \\
([0.3,0.2], 0.4), \\
([0.2,0.1], 0.1)\end{array}\right)$ \\
\hline$A_{4}$ & $\left(\begin{array}{c}([0.2,0.5], 0.3), \\
([0.3,0.1], 0.3), \\
([0.1,0.1], 0.2)\end{array}\right)$ & $\left(\begin{array}{c}([0.4,0.4], 0.4), \\
([0.3,0.2], 0.1), \\
([0.1,0.2], 0.1)\end{array}\right)$ & $\left(\begin{array}{c}([0.5,0.3], 0.3), \\
([0.2,0.1], 0.2), \\
([0.1,0.2], 0.2)\end{array}\right)$ \\
\hline
\end{tabular}

Step 1. We will use the concept of SCFWG operator to aggregate all spherical cubic fuzzy decision matrices that are normalized separately. The aggregated of spherical cubic fuzzy matrix is shown in Table 7.

Step 2

Case 1. We will use SCFDWA to evaluate their efficiency separately according to the weight vectors $\gamma=(0.2,0.3,0.5)^{T}$ and $\varepsilon=0.5>0$ shown in Table 8

Case 2. We will use SCFDOWA to evaluate their efficiency separately according to the weight vectors $\gamma=(0.2,0.3,0.5)^{T}$ and $\varepsilon=0.5>0$ shown in Table 9 Case 3. We will use SCFDHWA to evaluate their efficiency separately according to the weight vectors $\gamma=(0.2,0.3,0.5)^{T}$ and $\varepsilon=0.5>0$ shown in Table 10 
TABLE 4: Normalized investing capacity in a wealth administration $R^{1}$.

\begin{tabular}{|c|c|c|c|}
\hline & $b_{1}$ & $b_{2}$ & $b_{3}$ \\
\hline$A_{1}$ & $\left(\begin{array}{c}([0.5,0.4], 0.3), \\
([0.2,0.3], 0.1), \\
([0.2,0.2], 0.1)\end{array}\right)$ & $\left(\begin{array}{c}([0.3,0.4], 0.3), \\
([0.4,0.2], 0.1), \\
([0.2,0.1], 0.2)\end{array}\right)$ & $\left(\begin{array}{c}([0.4,0.5], 0.4), \\
([0.2,0.1], 0.2) \\
([0.4,0.1], 0.2)\end{array}\right)$ \\
\hline$A_{2}$ & $\left(\begin{array}{c}([0.1,0.6], 0.2), \\
([0.2,0.1], 0.2), \\
([0.4,0.2], 0.1)\end{array}\right)$ & $\left(\begin{array}{c}([0.4,0.1], 0.3), \\
([0.1,0.2], 0.3), \\
([0.3,0.4], 0.2)\end{array}\right)$ & $\left(\begin{array}{c}([0.4,0.3], 0.2), \\
([0.2,0.4], 0.3), \\
([0.2,0.3], 0.1)\end{array}\right)$ \\
\hline$A_{3}$ & $\left(\begin{array}{c}([0.2,0.4], 0.4), \\
([0.5,0.1], 0.2), \\
([0.1,0.4], 0.1)\end{array}\right)$ & $\left(\begin{array}{c}([0.1,0.1], 0.5), \\
([0.1,0.2], 0.1), \\
([0.3,0.2], 0.1)\end{array}\right)$ & $\left(\begin{array}{c}([0.4,0.3], 0.2) \\
([0.3,0.2], 0.1) \\
([0.2,0.4], 0.1)\end{array}\right)$ \\
\hline$A_{4}$ & $\left(\begin{array}{c}([0.4,0.3], 0.3), \\
([0.3,0.4], 0.3), \\
([0.2,0.1], 0.3)\end{array}\right)$ & $\left(\begin{array}{c}([0.4,0.4], 0.5), \\
([0.1,0.2], 0.1), \\
([0.1,0.1], 0.3)\end{array}\right)$ & $\left(\begin{array}{c}([0.5,0.4], 0.2), \\
([0.3,0.1], 0.2), \\
([0.1,0.2], 0.3)\end{array}\right)$ \\
\hline
\end{tabular}

TABLE 5: Normalized investing capacity in a wealth administration $R^{2}$.

\begin{tabular}{|c|c|c|c|}
\hline & $b_{1}$ & $b_{2}$ & $b_{3}$ \\
\hline$A_{1}$ & $\left(\begin{array}{c}([0.2,0.2], 0.1), \\
([0.5,0.3], 0.2), \\
([0.2,0.1], 0.4),\end{array}\right)$ & $\left(\begin{array}{c}([0.4,0.3], 0.2), \\
([0.3,0.1], 0.4), \\
([0.1,0.2], 0.2)\end{array}\right)$ & $\left(\begin{array}{c}([0.6,0.4], 0.3), \\
([0.2,0.3], 0.1), \\
([0.1,0.2], 0.2)\end{array}\right)$ \\
\hline$A_{2}$ & $\left(\begin{array}{c}([0.6,0.2], 0.4), \\
([0.1,0.1], 0.3), \\
([0.2,0.1], 0.2)\end{array}\right)$ & $\left(\begin{array}{c}([0.1,0.3], 0.4), \\
([0.2,0.1], 0.2), \\
([0.2,0.1], 0.4)\end{array}\right)$ & $\left(\begin{array}{c}([0.7,0.2], 0.4), \\
([0.1,0.3], 0.4), \\
([0.2,0.1], 0.2)\end{array}\right)$ \\
\hline$A_{3}$ & $\left(\begin{array}{c}([0.3,0.4], 0.2), \\
([0.2,0.1], 0.3), \\
([0.1,0.2], 0.3)\end{array}\right)$ & $\left(\begin{array}{c}([0.4,0.2], 0.2), \\
([0.3,0.1], 0.3), \\
([0.2,0.2], 0.1)\end{array}\right)$ & $\left(\begin{array}{c}([0.4,0.5], 0.3) \\
([0.1,0.1], 0.2), \\
([0.3,0.2], 0.3)\end{array}\right)$ \\
\hline$A_{4}$ & $\left(\begin{array}{c}([0.2,0.4], 0.3), \\
([0.1,0.2], 0.1), \\
([0.4,0.1], 0.3)\end{array}\right)$ & $\left(\begin{array}{c}([0.6,0.1], 0.6), \\
([0.1,0.2], 0.1), \\
([0.3,0.1], 0.2)\end{array}\right)$ & $\left(\begin{array}{c}([0.2,0.1], 0.4,) \\
([0.3,0.4], 0.1) \\
([0.3,0.2], 0.1)\end{array}\right)$ \\
\hline
\end{tabular}

TABLE 6: Normalized investing capacity in a wealth administration $R^{3}$.

\begin{tabular}{|c|c|c|c|}
\hline & $b_{1}$ & $b_{2}$ & $b_{3}$ \\
\hline$A_{1}$ & $\left(\begin{array}{c}([0.3,0.2], 0.4), \\
([0.2,0.2], 0.1), \\
([0.1,0.4], 0.4)\end{array}\right)$ & $\left(\begin{array}{c}([0.3,0.4], 0.4), \\
([0.4,0.4], 0.2), \\
([0.2,0.4], 0.2)\end{array}\right)$ & $\left(\begin{array}{c}([0.6,0.4], 0.1), \\
([0.2,0.3], 0.2), \\
([0.1,0.3], 0.1)\end{array}\right)$ \\
\hline$A_{2}$ & $\left(\begin{array}{c}([0.3,0.5], 0.2), \\
([0.1,0.1], 0.2), \\
([0.4,0.2], 0.1)\end{array}\right)$ & $\left(\begin{array}{c}([0.4,0.5], 0.1), \\
([0.1,0.5], 0.3), \\
([0.3,0.1], 0.2)\end{array}\right)$ & $\left(\begin{array}{c}([0.8,0.5], 0.2), \\
([0.1,0.3], 0.1), \\
([0.1,0.2], 0.1)\end{array}\right)$ \\
\hline$A_{3}$ & $\left(\begin{array}{c}([0.1,0.3], 0.2), \\
([0.2,0.1], 0.2)\end{array}\right)$ & $\left(\begin{array}{c}([0.1,0.3], 0.2), \\
([0.1,0.4], 0.3), \\
([0.3,0.1], 0.3)\end{array}\right)$ & $\left(\begin{array}{c}([0.2,0.1], 0.3), \\
([0.4,0.2], 0.3), \\
([0.1,0.1], 0.2)\end{array}\right)$ \\
\hline$A_{4}$ & $\left(\begin{array}{c}([0.3,0.5], 0.2), \\
([0.3,0.1], 0.3), \\
([0.2,0.1], 0.1)\end{array}\right)$ & $\left(\begin{array}{c}([0.4,0.4], 0.4), \\
([0.1,0.2], 0.3), \\
([0.1,0.2], 0.1)\end{array}\right)$ & $\left(\begin{array}{c}([0.3,0.3], 0.5), \\
([0.2,0.1], 0.2), \\
([0.2,0.2], 0.1)\end{array}\right)$ \\
\hline
\end{tabular}

Case 4. We will use SCFDWG to evaluate their efficiency separately according to the weight vectors $\gamma=(0.2,0.3,0.5)^{T}$ and $\varepsilon=0.5>0$ shown in Table 11 Case 5. We will use SCFDOWG to evaluate their efficiency separately according to the weight vectors $\gamma=(0.2,0.3,0.5)^{T}$ and $\varepsilon=0.5>0$ shown in Table 12
Case 6 We will use SCFDHWG to evaluate their efficiency separately according to the weight vectors $\gamma=(0.2,0.3,0.5)^{T}$ and $\varepsilon=0.5>0$ shown in Table 13

Step 3. The score of each alternative is shown in Table 14. 
TABLe 7: Aggregated spherical cubic fuzzy decision information matrix R.

\begin{tabular}{|c|c|c|c|}
\hline & $b_{1}$ & $b_{2}$ & $b_{3}$ \\
\hline$A_{1}$ & $\left(\begin{array}{c}([0.2056,0.1469], 0.1852), \\
([0.1537,0.158], 0.1), \\
([0.1,0.1948], 0.2505)\end{array}\right)$ & $\left(\begin{array}{c}([0.2268,0.2646], 0.1852), \\
([0.2646,0.1453], 0.1406), \\
([0.1,0.1948], 0.1)\end{array}\right)$ & $\left(\begin{array}{c}([0.4181,0.3318], 0.1821), \\
([0.1,0.1569], 0.1), \\
([0.1969,0.1425], 0.1)\end{array}\right)$ \\
\hline$A_{2}$ & $\left(\begin{array}{c}([0.2085,0.2814], 0.1406), \\
([0.1,0.1], 0.124), \\
([0.2567,0.1], 0.1)\end{array}\right)$ & $\left(\begin{array}{c}([0.2134,0.1986], 0.1792), \\
([0.1,0.1602], 0.1613), \\
([0.1755,0.1969], 0.1885)\end{array}\right)$ & $\left(\begin{array}{c}([0.4961,0.2042], 0.1406), \\
([0.1,0.2305], 0.1792), \\
([0.1,0.1435], 0.1)\end{array}\right)$ \\
\hline$A_{3}$ & $\left(\begin{array}{c}([0.2143,0.3], 0.2353), \\
([0.1625,0.1266], 0.124), \\
([0.1,0.1969], 0.1393)\end{array}\right)$ & $\left(\begin{array}{c}([0.1406,0.1266], 0.625), \\
([0.1240,0.1453], 0.1569), \\
([0.1755,0.1], 0.1425)\end{array}\right)$ & $\left(\begin{array}{c}([0.2065,0.1959], 0.1569), \\
([0.1852,0.1], 0.1266), \\
([0.1393,0.1969], 0.1393)\end{array}\right)$ \\
\hline$A_{4}$ & $\left(\begin{array}{c}([0.1859,0.2871], 0.158), \\
([0.1613,0.1469], 0.1613), \\
([0.1885,0.1], 0.1729)\end{array}\right)$ & $\left(\begin{array}{c}([0.3515,0.2134], 0.3887), \\
([0.1,0.1], 0.1266), \\
([0.1392,0.1], 0.1435)\end{array}\right)$ & $\left(\begin{array}{c}([0.2056,0.1859], 0.2252), \\
([0.158,0.1406], 0.1), \\
([0.1393,0.1], 0.435)\end{array}\right)$ \\
\hline
\end{tabular}

TABle 8: Aggregated spherical cubic data (SCFDWA).

\begin{tabular}{|c|c|}
\hline & $(([0.2587,0.213], 0.1856))$, \\
\hline$A_{1}$ & $\left(\begin{array}{c}([0.9859,0.988], 0.994), \\
([0.9927,0.983], 0.981)\end{array}\right)$ \\
\hline$A_{2}$ & $\left(\begin{array}{c}([0.2782,0.25], 0.1494), \\
([0.9949,0.99], 0.9896), \\
([0.9777,0.992], 0.9929),\end{array}\right)$ \\
\hline$A_{3}$ & $\left(\begin{array}{c}([0.1988,0.246], 0.2057), \\
([0.9871,0.992], 0.9912), \\
([0.9922,0.984], 0.99),\end{array}\right)$ \\
\hline$A_{4}$ & $\left(\begin{array}{c}([0.2271,0.253], 0.224), \\
([0.9888,0.991], 0.9898), \\
([0.9856,0.995], 0.9868)\end{array}\right.$ \\
\hline
\end{tabular}

TABle 9: Aggregated spherical cubic data (SCFDOWA).

$\begin{array}{lll}A_{1} & & \left(\begin{array}{c}([0.4161,0.373], 0.2874), \\ ([0.9636,0.961], 0.9883) \\ ([0.9812,0.964], 0.9774)\end{array}\right) \\ & \left(\begin{array}{c}(0.4865,0.321], 0.2467), \\ ([0.9918,0.965], 0.972), \\ ([0.972,0.976], 0.9849)\end{array}\right) \\ A_{2} & \left(\begin{array}{c}([0.3106,0.344], 0.3407), \\ ([0.9798,0.987], 0.9792), \\ ([0.9765,0.967], 0.9781)\end{array}\right) \\ A_{3} & \left(\begin{array}{c}(0.3977,0.274], 0.3784), \\ ([0.9858,0.976], 0.9818), \\ ([0.9743,0.989], 0.9749)\end{array}\right) \\ & A_{4} & \end{array}$

Step 4. By using the rank criteria $b_{i}(i=1,2, \ldots, m)$, we will select the best one which has the largest score.

\section{Comparative Analysis}

Here, we provide two comparison analysis that show that our proposed operators reliable and effective to aggregate the spherical cubic data. In Figure 1, ranking of spherical cubic fuzzy Dombi operators is given (Tables 14-20).
In 2019, Jana et al. suggested the picture fuzzy Dombi aggregation operators; in this study, we give comparison between proposed spherical cubic Dombi aggregation operators and existed Dombi aggregation operators as follows.

Now, utilizing spherical cubic fuzzy Dombi weighted averaging operator to chose the best alternative is as follows as follows.

$\check{Q}_{1}$ is the optimal alternative. The findings are similar to those provided by Jana et al. [25]. The methodology 
TABLE 10: Aggregated spherical cubic data (SCFDHWA).

\begin{tabular}{ll}
\hline$A_{1}$ & $\left(\begin{array}{c}([0.4954,0.39], 0.2529), \\
([0.9624,0.958], 0.9867), \\
([0.9817,0.964], 0.975)\end{array}\right)$ \\
$A_{2}$ & $\left(\begin{array}{c}([0.5013,0.342], 0.2631), \\
([0.9899,0.959], 0.9686), \\
([0.9684,0.971], 0.983)\end{array}\right)$ \\
$A_{3}$ & $\left(\begin{array}{c}([0.3303,0.368], 0.3559), \\
([0.9777,0.985], 0.9761) \\
([0.9738,0.964], 0.9735)\end{array}\right)$ \\
$A_{4}$ & $\left(\begin{array}{c}([0.3905,0.272], 0.3587), \\
([0.9801,0.981], 0.9828), \\
([0.9699,0.99], 0.9725)\end{array}\right)$ \\
\hline
\end{tabular}

Table 11: Aggregated spherical cubic data (SCFDWG).

\begin{tabular}{ll}
\hline$A_{1} \quad$ & $\left(\begin{array}{c}([0.9767,0.9873], 0.989), \\
([0.1672,0.156], 0.109), \\
([0.121,0.1852], 0.1911)\end{array}\right)$ \\
$A_{2}$ & $\left(\begin{array}{c}([0.9714,0.9773], 0.9936), \\
([0.1006,0.1407], 0.144), \\
([0.2101,0.13], 0.1192)\end{array}\right)$ \\
$A_{3}$ & $\left(\begin{array}{c}([0.9853,0.9751], 0.9848), \\
([0.1602,0.1257], 0.1321), \\
([0.1245,0.1783], 0.1407)\end{array}\right)$ \\
$A_{4} \quad\left(\begin{array}{c}([0.9881,0.9774], 0.99), \\
([0.149,0.1368], 0.1425) \\
([0.1693,0.1006], 0.1618)\end{array}\right)$ \\
\hline
\end{tabular}

presented by Jana et al. [25] tackles picture fuzzy set and fails to deal spherical cubic fuzzy set. Therefore, the new method proposed in this paper is more generalized to deal uncertainties in decision-making problems. Therefore, novel spherical cubic Dombi aggregation operators are more efficient and accurate in solving group decision-making problems compared with existing Dombi aggregation operators.

Ashraf et al. [5] suggested the spherical fuzzy Dombi aggregated operations to aggregate the spherical fuzzy numbers; in the present section, we give the comparison between proposed and novel spherical cubic Dombi aggregated operators. We get the spherical Dombi information from Ashraf et al. [5] as follows.

The best alternative is $A_{2}$.

Now, to utilize spherical cubic fuzzy Dombi weighted averaging operator to chose the best alternative is as follows.

The best choice is $A_{4}$. Results are similar to Ashraf et al. [5]. The methodology proposed by Ashraf et al. [5] dealt with spherical fuzzy set, and we extend it for spherical cubic fuzzy set to get more accurate results. The methodology presented
TABLE 12: Aggregated spherical cubic data (SCFDOWG).

\begin{tabular}{ll}
\hline$A_{1} \quad$ & $\left(\begin{array}{c}([0.9093,0.9278], 0.9578), \\
([0.2674,0.275], 0.1525), \\
([0.1929,0.2675], 0.2112)\end{array}\right)$ \\
$A_{2}$ & $\left(\begin{array}{c}([0.8737,0.9471], 0.9691), \\
([0.1275,0.2616], 0.2352), \\
([0.2351,0.2176], 0.1732)\end{array}\right)$ \\
$A_{3}$ & $\left(\begin{array}{c}([0.9505,0.9389], 0.9402), \\
([0.1999,0.1618], 0.2029), \\
([0.2154,0.2539], 0.208)\end{array}\right)$ \\
$A_{4}$ & $\left(\begin{array}{c}([0.9175,0.9619], 0.9257), \\
([0.1678,0.2178], 0.19), \\
([0.2253,0.1495], 0.2225)\end{array}\right)$ \\
\hline
\end{tabular}

TABLE 13: Aggregated spherical cubic data (SCFDHWG).

\begin{tabular}{ll}
$A_{1}$ & $\left(\begin{array}{c}([0.8686,0.9207], 0.9675), \\
([0.2715,0.2851], 0.1623), \\
([0.1902,0.2665], 0.2221)\end{array}\right)$ \\
$A_{2}$ & $\left(\begin{array}{c}([0.8653,0.9396], 0.9648), \\
([0.1416,0.2820], 0.2487), \\
([0.2493,0.2383], 0.1836)\end{array}\right)$ \\
$A_{3}$ & $\left(\begin{array}{c}([0.9439,0.9299], 0.9345), \\
([0.2098,0.1745], 0.2172), \\
([0.2272,0.2675], 0.2287)\end{array}\right)$ \\
$A_{4}$ & $\left(\begin{array}{c}([0.9206,0.9624], 0.9335), \\
([0.1985,0.1937], 0.1846), \\
([0.2435,0.1386], 0.2329)\end{array}\right)$ \\
\hline
\end{tabular}

TABle 14: Ranking by spherical cubic Dombi aggregation operators.

\begin{tabular}{lccccc}
\hline & $A_{1}$ & $A_{2}$ & $A_{3}$ & $A_{4}$ & Ranking \\
\hline SCFDWA & 0.0564 & 0.0493 & 0.055 & 0.0593 & $A_{4}>A_{1}>A_{3}>A_{2}$ \\
SCFDOWA & 0.1257 & 0.1209 & 0.123 & 0.1261 & $A_{4}>A_{1}>A_{3}>A_{2}$ \\
SCFDHWA & 0.1357 & 0.1231 & 0.133 & 0.1414 & $A_{4}>A_{1}>A_{3}>A_{2}$ \\
SCFDWG & 0.9622 & 0.955 & 0.9614 & 0.9702 & $A_{4}>A_{1}>A_{3}>A_{2}$ \\
SCFDOWG & 0.8715 & 0.8646 & 0.8714 & 0.8740 & $A_{4}>A_{1}>A_{3}>A_{2}$ \\
SCFDHWG & 0.8583 & 0.8506 & 0.8525 & 0.8763 & $A_{4}>A_{1}>A_{3}>A_{2}$ \\
\hline
\end{tabular}

for this article is more comprehensive to tackle the vagueness in decision-making problems. Consequently, spherical cubic Dombi aggregated operations are more reliable and effective to comprehend the decision issues when contrasted to existed Dombi aggregated operations.

The obtained results by using the concept of spherical cubic fuzzy Dombi aggregated operations give nearest results of ranking of spherical Dombi aggregated operations, and more suitable and accurate results of decision-making problems are given in Figure 2. 


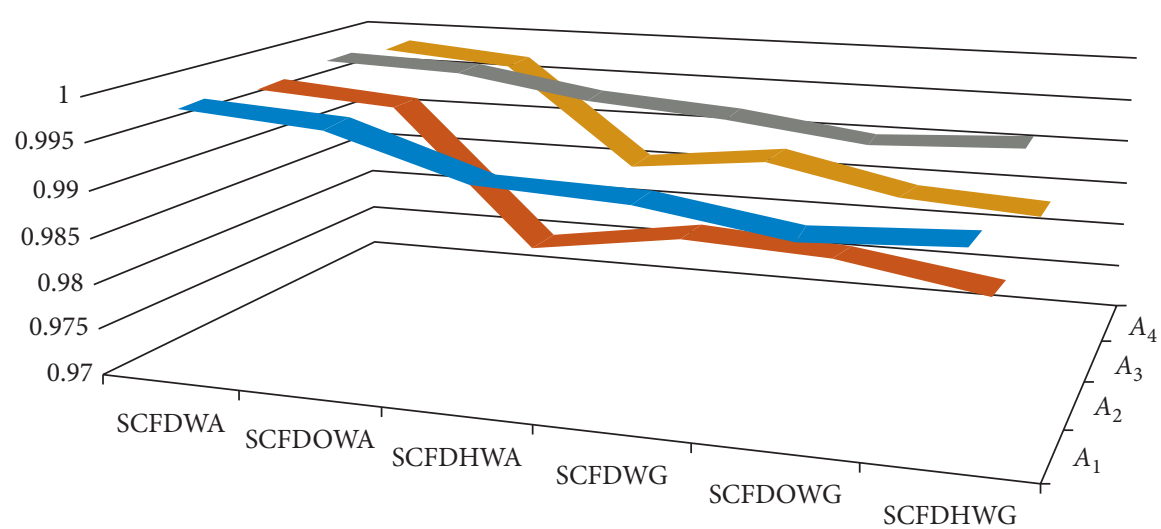

\begin{tabular}{|c|c|c|c|c|c|c|}
\hline & SCFDWA & SCFDOWA & SCFDHWA & SCFDWG & SCFDOWG & SCFDHWG \\
\hline$A_{1}$ & 0.99823 & 0.9972 & 0.9925 & 0.9918 & 0.9895 & 0.9904 \\
\hline$A_{2}$ & 0.998 & 0.9969 & 0.9824 & 0.985 & 0.984 & 0.9816 \\
\hline$A_{3}$ & 0.9989 & 0.9982 & 0.996 & 0.9948 & 0.9929 & 0.9938 \\
\hline$A_{4}$ & 0.9982 & 0.997 & 0.9853 & 0.9873 & 0.984 & 0.9831 \\
\hline
\end{tabular}

FIgURE 1: Ranking using spherical cubic fuzzy dombi operator.

TABLE 15: Ranking criteria.

\begin{tabular}{lr}
\hline & \multicolumn{1}{c}{ Ranking } \\
\hline SCFDWA & $A_{4}>A_{1}>A_{3}>A_{2}$ \\
SCFDOWA & $A_{4}>A_{1}>A_{3}>A_{2}$ \\
SCFDHWA & $A_{4}>A_{1}>A_{3}>A_{2}$ \\
SCFDWG & $A_{4}>A_{1}>A_{3}>A_{2}$ \\
SCFDOWG & $A_{4}>A_{1}>A_{3}>A_{2}$ \\
SCFDHWG & $A_{4}>A_{1}>A_{3}>A_{2}$ \\
\hline
\end{tabular}

Table 16: Picture fuzzy matrix (Jana et al. 2019).

\begin{tabular}{ll}
\hline$\check{Q}_{1}$ & $(0.56,0.34,0.10)(0.90,0.07,0.03)(0.40,0.33,0.19)(0.09,0.79,0.03)$ \\
$\check{Q}_{2}$ & $(0.70,0.10,0.09)(0.10,0.66,0.20)(0.06,0.81,0.12)(0.72,0.14,0.09)$ \\
$\check{Q}_{3}$ & $(0.88,0.09,0.03)(0.08,0.10,0.06)(0.05,0.83,0.09)(0.65,0.25,0.07)$ \\
$\check{Q}_{4}$ & $(0.80,0.07,0.04)(0.70,0.15,0.11)(0.03,0.88,0.05)(0.07,0.82,0.05)$ \\
$\check{Q}_{5}$ & $(0.85,0.06,0.03)(0.64,0.07,0.22)(0.06,0.88,0.05)(0.13,0.77,0.09)$ \\
\hline
\end{tabular}

TABLE 17: Ranking by picture fuzzy aggregation operators.

\begin{tabular}{lllllll}
\hline$\rho$ & $S_{c}\left(\check{Q}_{1}\right)$ & $S_{c}\left(\check{Q}_{2}\right)$ & $S_{c}\left(\check{Q}_{3}\right)$ & $S_{c}\left(\check{Q}_{4}\right)$ & $S_{c}\left(\check{Q}_{5}\right)$ & Ranking \\
\hline 1 & 0.9977 & 0.9935 & 0.9967 & 0.9975 & 0.9887 & $\check{Q}_{1}>\check{Q}_{4}>\check{Q}_{3}>\check{Q}_{2}>\check{Q}_{5}$ \\
2 & 0.9978 & 0.9931 & 0.9963 & 0.9971 & 0.9878 & $Q_{1}>Q_{4}>Q_{3}>Q_{2}>Q_{5}$ \\
3 & 0.9976 & 0.9931 & 0.9962 & 0.9975 & 0.9877 & $\check{Q}_{1}>\check{Q}_{4}>\check{Q}_{3}>\check{Q}_{2}>\check{Q}_{5}$ \\
4 & 0.9976 & 0.9932 & 0.9961 & 0.9975 & 0.9877 & $Q_{1}>\mathscr{Q}_{4}>Q_{3}>\mathscr{Q}_{2}>Q_{5}$ \\
5 & 0.9975 & 0.9933 & 0.9960 & 0.9975 & 0.9879 & $\check{Q}_{1}>\check{Q}_{4}>\check{Q}_{3}>\check{Q}_{2}>\check{Q}_{5}$ \\
6 & 0.9975 & 0.9933 & 0.9961 & 0.9975 & 0.9880 & $\check{Q}_{1}>\check{Q}_{4}>\check{Q}_{3}>\check{Q}_{2}>\check{Q}_{5}$ \\
\hline
\end{tabular}

Table 18: Collective spherical Dombi fuzzy information matrix (Ashraf et al. 202).

\begin{tabular}{ll}
\hline$A_{1}$ & $(0.6582,0.4279,0.2947)(0.5742,0.3611,0.3398)(0.6297,0.4954,0.4093)$ \\
$A_{2}$ & $(0.7339,0.4891,0.2905)(0.4523,0.6776,0.2498)(0.6582,0.3076,0.4993)$ \\
$A_{3}$ & $(0.5134,0.5334,0.3894)(0.6844,0.2763,0.2739)(0.6236,0.2667,0.2731)$ \\
$A_{4}$ & $(0.6435,0.3934,0.2715)(0.4954,0.2445,0.4523)(0.6603,0.2223,0.4353)$ \\
\hline
\end{tabular}


TABLE 19: Ranking of alternatives through Dombi aggregation operators (Ashraf et al. 2020).

\begin{tabular}{lccccc}
\hline & $A_{1}$ & $A_{2}$ & $A_{3}$ & $A_{4}$ & Ranking \\
\hline SFDWA & 0.8242 & 0.8936 & 0.8845 & 0.8809 & $A_{2}>A_{3}>A_{4}>A_{1}$ \\
SFDOWA & 0.8245 & 0.8905 & 0.8853 & 0.8537 & $A_{2}>A_{3}>A_{4}>A_{1}$ \\
SFDHWA & 0.8653 & 0.9757 & 0.8734 & 0.8731 & $A_{2}>A_{3}>A_{4}>A_{1}$ \\
SFDWG & 0.3930 & 0.4427 & 0.3770 & 0.3518 & $A_{2}>A_{1}>A_{3}>A_{4}$ \\
SFDOWG & 0.4393 & 0.4532 & 0.4374 & 0.3674 & $A_{2}>A_{1}>A_{3}>A_{4}$ \\
SFDHWG & 0.7945 & 0.9044 & 0.7335 & 0.7163 & $A_{2}>A_{1}>A_{3}>A_{4}$ \\
\hline
\end{tabular}

TABle 20: Ranking of alternatives by spherical cubic Dombi aggregation operators.

\begin{tabular}{lccccc}
\hline & $A_{1}$ & $A_{2}$ & $A_{3}$ & $A_{4}$ & Ranking \\
\hline SCFDWA & 0.0564 & 0.0493 & 0.055 & 0.0593 & $A_{4}>A_{1}>A_{3}>A_{2}$ \\
SCFDOWA & 0.1257 & 0.1209 & 0.123 & 0.1261 & $A_{4}>A_{1}>A_{3}>A_{2}$ \\
SCFDHWA & 0.1357 & 0.1231 & 0.133 & 0.1414 & $A_{4}>A_{1}>A_{3}>A_{2}$ \\
SCFDWG & 0.9622 & 0.955 & 0.9614 & 0.9702 & $A_{4}>A_{1}>A_{3}>A_{2}$ \\
SCFDOWG & 0.8715 & 0.8646 & 0.8714 & 0.8740 & $A_{4}>A_{1}>A_{3}>A_{2}$ \\
SCFDHWG & 0.8583 & 0.8506 & 0.8525 & 0.8763 & $A_{4}>A_{1}>A_{3}>A_{2}$ \\
\hline
\end{tabular}

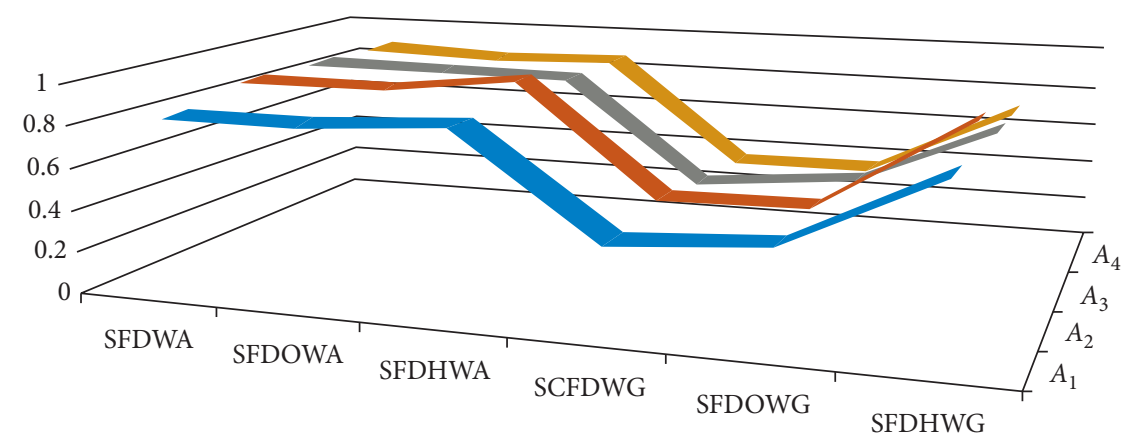

\begin{tabular}{|c|c|c|c|c|c|c|}
\cline { 2 - 7 } \multicolumn{1}{c|}{} & SFDWA & SFDOWA & SFDHWA & SFDWG & SFDOWG & SFDHWG \\
\hline$A_{1}$ & 0.8242 & 0.8245 & 0.8653 & 0.393 & 0.4393 & 0.7945 \\
\hline$A_{2}$ & 0.8936 & 0.8905 & 0.9757 & 0.4427 & 0.4532 & 0.9044 \\
\hline$A_{3}$ & 0.8845 & 0.8853 & 0.8734 & 0.377 & 0.4374 & 0.7335 \\
\hline$\square A_{4}$ & 0.8509 & 0.8537 & 0.8731 & 0.3518 & 0.3674 & 0.7163 \\
\hline \multicolumn{7}{|c|}{} \\
\end{tabular}

FIgURE 2: Ranking using spherical cubic fuzzy Dombi operator.

\section{Conclusion}

In this paper, the concept of spherical cubic fuzzy set is introduced that is the generalization of the spherical fuzzy set. Further, some spherical cubic fuzzy operational laws are established. Moreover, score and accuracy functions are defined for the comparison of spherical cubic fuzzy numbers. In addition, the idea for Dombi aggregated operations of spherical cubic fuzzy set is presented. Furthermore, the fundamental characteristics of spherical cubic fuzzy Dombi aggregated operations are presented. For the aggregation of spherical cubic fuzzy sets, we proposed SCFDWA, SCFDOWA, SCFDHWA, SCFDWG, SCFDOWG, and SCFDHWG under the spherical cubic fuzzy information. Additionally, some properties like idempotency, boundary, and monotonicity are discussed, and a relation between these established operators is shown. Likewise, a multiattribute decision-making methodology to illustrate the efficiency of the proposed operators is suggested. In addition, we applied the developed aggregation operators to discuss the decision-making problems. A numerical illustration was proposed to demonstrate the efficiency of the suggested operators over alternate methods in decisionmaking problems. Finally, to determine the validity and efficiency of the novel approach, we carried out the comparative analysis among the existing and the proposed operators.

In future, we will be integrating other approaches with SCFSs like Einstein sum and product to develop the ideas of spherical cubic fuzzy Einstein weighted averaging (SCFEWA), spherical cubic fuzzy Einstein ordered weighted averaging (SCFEOWA), spherical cubic fuzzy Einstein hybrid weighted averaging (SCFEHWA), spherical cubic fuzzy Einstein weighted geometric (SCFEWG), spherical cubic 
fuzzy Einstein ordered weighted geometric (SCFEOWG), spherical cubic fuzzy Einstein hybrid weighted geometric (SCFEHWG), and more generalized operators in multiattribute decision-making problems. We can extend these defined Dombi aggregation operators for Hamacher and Frank norms to deal with uncertainty in the data by using spherical cubic fuzzy information. We can extend this work for power aggregation operators, Dombi Bonferroni mean operators, and their application to multiattribute group decision-making. The application of our proposed model in the future may be used in decision-making theory, risk analysis, and other domains in uncertain environments.

\section{Data Availability}

No data were used to support this study.

\section{Conflicts of Interest}

The authors declare that they have no conflicts of interest.

\section{Acknowledgments}

The authors are grateful to the Deanship of Scientific Research, King Saud University, for funding through Vice Deanship of Scientific Research Chairs.

\section{References}

[1] L. A. Zadeh, "Fuzzy sets," Information and Control, vol. 8, no. 3, pp. 338-353, 1965.

[2] K. T. Atanassov, "Intuitionistic fuzzy sets," Fuzzy Sets and Systems, vol. 20, no. 1, pp. 87-96, 1986.

[3] R. R. Yager, "Pythagorean fuzzy subsets," in Proceedings of the 2013 joint IFSA world congress and NAFIPS annual meeting (IFSA/NAFIPS), pp. 57-61, IEEE, Edmonton, Canada, June 2013.

[4] R. R. Yager, "Pythagorean membership grades in multicriteria decision making," IEEE Trans Fuzzy Syst, vol. 22, no. 4, pp. 958-965, 2013b.

[5] S. Ashraf, S. Abdullah, and T. Mahmood, "Spherical fuzzy Dombi aggregation operators and their application in group decision making problems," Journal of Ambient Intelligence and Humanized Computing, vol. 11, no. 7, pp. 2731-2749, 2019.

[6] S. Ashraf, T. Mahmood, S. Abdullah, and Q. Khan, "Different approaches to multi-criteria group decision making problems for picture fuzzy environment," Bulletin of the Brazilian Mathematical Society, New Series, vol. 50, no. 2, pp. 373-397, 2018a.

[7] B. C. Cuong, Picture Fuzzy Sets-First Results, Part 1. Seminar Neuro-Fuzzy Systems with Applications, Institute of Mathematics, Hanoi, Vietnam, 2013a.

[8] B. C. Cuong, Picture Fuzzy Sets-First Results, Part 2. Seminar Neuro-Fuzzy Systems with Applications, Institute of Mathematics, Hanoi, 2013b.

[9] B. C. Cuong, "Picture fuzzy sets," Journal of Computer Science and Cybernetics, vol. 30, no. 4, pp. 409-420, 2014.

[10] S. Ashraf and S. Abdullah, "Spherical aggregation operators and their application in multiattribute group decision-making," International Journal of Intelligent Systems, vol. 34, no. 3, pp. 493-523, 2019.
[11] A. Fahmi, F. Amin, S. Abdullah, and M. Shakeel, "Power average operators of trapezoidal cubic fuzzy numbers and application to multi-attribute group decision making," Journal of Intelligent Systems, vol. 29, no. 1, pp. 1643-1661, 2020.

[12] H. Garg, "Some picture fuzzy aggregation operators and their applications to multicriteria decision-making," Arabian Journal for Science and Engineering, vol. 42, no. 12, pp. 5275-5290, 2017.

[13] S. Ashraf, S. Abdullah, M. Aslam, M. Qiyas, and M. A. Kutbi, "Spherical fuzzy sets and its representation of spherical fuzzy t-norms and t-conorms," Journal of Intelligent \& Fuzzy Systems, vol. 36, no. 6, pp. 6089-6102, 2019.

[14] K. F. Gündogdu and C. Kahraman, "Spherical fuzzy sets and spherical fuzzy TOPSIS method," Journal of Intelligent and Fuzzy Systems, vol. 36, no. 1, pp. 337-352, 2019.

[15] J. Chen and J. Ye, "Some single-valued neutrosophic dombi weighted aggregation operators for multiple attribute decision-making," Symmetry, vol. 9, no. 6, p. 82, 2017.

[16] P. Liu, J. Liu, and S.-M. Chen, "Some intuitionistic fuzzy Dombi Bonferroni mean operators and their application to multi-attribute group decision making," Journal of the Operational Research Society, vol. 69, no. 1, pp. 1-24, 2018.

[17] P. Liu, B. Zhu, and P. Wang, "A multi-attribute decisionmaking approach based on spherical fuzzy sets for Yunnan baiyao's R\&D project selection problem," International Journal of Fuzzy Systems, vol. 21, no. 7, pp. 2168-2191, 2019.

[18] L. Shi and J. Ye, "Dombi aggregation operators of neutrosophic cubic sets for multiple attribute decision-making," Algorithms, vol. 11, no. 3, p. 29, 2018.

[19] X. Lu and J. Ye, "Dombi aggregation operators of linguistic cubic variables for multiple attribute decision making," Information, vol. 9, no. 8, p. 188, 2018.

[20] A. Khan, S. Ashraf, S. Abdullah, M. Qiyas, J. Luo, and S. Khan, "Pythagorean fuzzy Dombi aggregation operators and their application in decision support system," Symmetry, vol. 11, no. 3, p. 383, 2019.

[21] Q. Khan, P. Liu, T. Mahmood, F. Smarandache, and K. Ullah, "Some interval neutrosophic dombi power bonferroni mean operators and their application in multi-attribute decisionmaking," Symmetry, vol. 10, no. 10, p. 459, 2018.

[22] G. Wei and Y. Wei, "Some single-valued neutrosophic Dombi prioritized weighted aggregation operators in multiple attribute decision making," Journal of Intelligent and Fuzzy Systems, vol. 9, no. 8, pp. 1-3, 2018.

[23] H. Zhang, R. Zhang, H. Huang, and J. Wang, "Some picture fuzzy dombi heronian mean operators with their application to multi-attribute decision-making," Symmetry, vol. 10, no. 11, p. 593, 2018.

[24] C. Jana, M. Pal, and J. Q. Wang, "Bipolar fuzzy Dombi aggregation operators and its application in multiple-attribute decision making process," Journal of Ambient Intelligence and Humanized Computing, vol. 10, pp. 3533-3549, 2018.

[25] C. Jana, T. Senapati, M. Pal, and R. R. Yager, "Picture fuzzy Dombi aggregation operators: application to MADM process," Applied Soft Computing, vol. 74, pp. 99-109, 2019.

[26] M. Rafiq, S. Ashraf, S. Abdullah, T. Mahmood, and S. Muhammad, "The cosine similarity measures of spherical fuzzy sets and their applications in decision making," Journal of Intelligent \& Fuzzy Systems, vol. 36, no. 8, 2019.

[27] R. Seikh and U. Mandal, "Intuitionistic fuzzy Dombi aggregation operators and their application to multiple attribute decision-making," Granular Computing, vol. 10, 2019. 
[28] G. Wei, J. Wang, M. Lu, J. Wu, and C. Wei, "Similarity measures of spherical fuzzy sets based on cosine function and their applications," IEEE Access, vol. 7, pp. 159069-159080, 2019.

[29] A. S. Muneeza, S. Abdullah, and M. Aslam, "New multicriteria group decision support systems for small hydropower plant locations selection based on intuitionistic cubic fuzzy aggregation information," International Journal of Intelligent Systems, vol. 35, no. 6, pp. 983-1020, 2020.

[30] A. S. Muneeza, "Multicriteria group decision making for supplier selection based on intuitionistic cubic fuzzy aggregation operators," International Journal of Fuzzy Systems, vol. 22, pp. 810-823, 2020.

[31] M. S. A. Khan, F. Khan, J. Lemley, S. Abdullah, and F. Hussain, "Extended topsis method based on Pythagorean cubic fuzzy multi-criteria decision making with incomplete weight information," Journal of Intelligent \& Fuzzy Systems, vol. 38, no. 2, pp. 2285-2296, 2020.

[32] F. Khan, S. Abdullah, T. Mahmood, M. Shakeel, M. Rahim, and N. ul Amin, "Pythagorean cubic fuzzy aggregation information based on confidence levels and its application to multi-criteria decision making process," Journal of Intelligent \& Fuzzy Systems, vol. 36, no. 6, pp. 5669-5683, 2019.

[33] F. Khan, M. S. A. Khan, M. Shahzad, and S. Abdullah, "Pythagorean cubic fuzzy aggregation operators and their application to multi-criteria decision making problems," Journal of Intelligent \& Fuzzy Systems, vol. 36, no. 1, pp. 595-607, 2019.

[34] T. Ayaz, M. M. Al-Shomrani, S. Abdullah, and A. Hussain, "Evaluation of enterprise production based on spherical cubic hamacher aggregation operators," Mathematics, vol. 8, no. 10, p. 1761, 2020.

[35] H. A. Tehreem and M. S. A. Khan, "Average operators based on spherical cubic fuzzy number and their application in multi-attribute decision making," Annals of Optimization Theory and Practice, vol. 3, no. 4, pp. 83-111, 2020. 